\title{
Neuro-adaptive Fixed-time Non-singular Fast Terminal Sliding Mode Control Design for a Class of Under-actuated Nonlinear Systems
}

SAFEER ULLAH ( $\sim$ safeer_iiui@yahoo.com )

COMSATS University Islamabad https://orcid.org/0000-0001-8017-7006

\section{Qudrat Khan}

COMSATS University Islamabad

Adeel Mehmood

COMSATS University Islamabad

\section{Research Article}

Keywords: Cart pendulum , Fixed-time stability , Quadcopter, RBF Neural network, Regular form , Terminal attractor, Under-actuated system

Posted Date: May 19th, 2021

DOl: https://doi.org/10.21203/rs.3.rs-494194/v1

License: (9) This work is licensed under a Creative Commons Attribution 4.0 International License. Read Full License

Version of Record: A version of this preprint was published at International Journal of Control on March 22nd, 2022. See the published version at https://doi.org/10.1080/00207179.2022.2056514. 


\title{
Neuro-adaptive fixed-time non-singular fast terminal sliding mode control design for a class of under-actuated nonlinear systems
}

\author{
Safeer Ullah • Qudrat Khan • Adeel \\ Mehmood
}

Received: date / Accepted: date

\begin{abstract}
This paper presents a fixed-time control design for a class of uncertain under-actuated nonlinear systems (UNS) using a non-singular fast terminal sliding mode control (TSMC) with a radial basis function (RBF) based estimator to achieve the fast convergence and robustness against the uncertain disturbances. The generalized mathematical model of the considered class is first reduced into an equivalent regular form, which is more convenient for any control synthesis design. A fast TSMC is designed for the transformed regular form to improve the control performance and annihilate the associated singularity problem of the conventional TSMC scheme. The steering of sliding manifold and system states in fixed-time is ensured through the Lyapunov stability theory. The RBF-based neural networks are used to adaptively estimate the nonlinear drift functions, which are feedbacked to the control input. The theoretical design, analysis and simulations of cart-pendulum and quadcopter systems demonstrate the feasibility and benefits of the regular form transformation and the designed control design. Comparing the proposed control synthesis with the standard literature presents the attractive nature of the proposed method for such a class.
\end{abstract}

Keywords Cart pendulum · Fixed-time stability · Quadcopter · RBF Neural network · Regular form - Terminal attractor · Under-actuated system

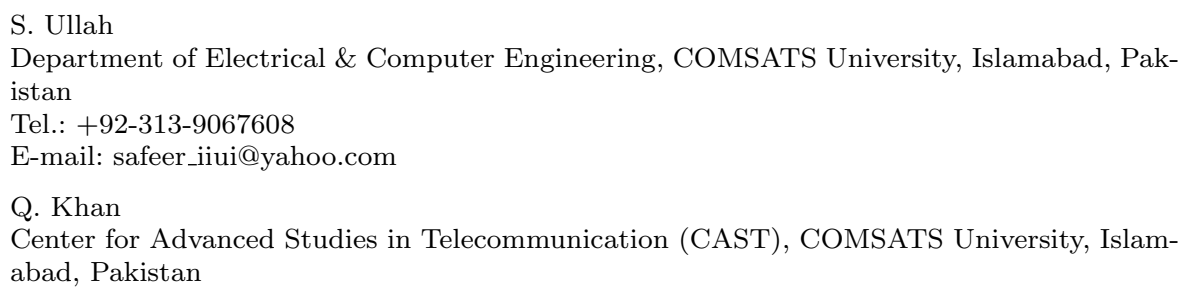

A. Mehmood

Department of Electrical \& Computer Engineering, COMSATS University, Islamabad, Pakistan 


\section{Introduction}

under-actuated systems are mechanical systems with less dimension of space spanned by the applied stabilizing inputs than space spanned by the configuration variables $[6,26]$. Because of the low probability of system failure, low power consumption, and low cost and weight, robust control of under-actuated mechanical systems is becoming increasingly important in today's research community, and they are now regularly used in a number of applications such as robotics, aerospace vehicles, and marine vehicles. This class also has several potential applications in locomotive systems, underwater vehicles, surface vessels, robots, satellites, and unmanned aerial vehicles. The stabilization of this class, however, is a difficult task due to high-order nonholonomic constraints, the lack of actuators for certain configuration variables, and coupling effects [28]. A large number of researchers synthesized various advanced nonlinear control algorithms to provide appealing results as well as closed-loop stability. Some of these efforts are detailed in this article.

Backstepping, a nonlinear control algorithm, is used to transform the $n^{t} h$ order structure into a new recursive form with $\mathrm{n}$ number of subsystems (each one of relative degree one). This control scheme has generally been used in recent years for the global stabilization of UNS, such as surface vessel [8], spacecraft [10] and unmanned aerial vehicle [9]. Unfortunately, as the degree of freedom of the aforementioned class increases, such a control strategy's design procedure becomes incredibly hard. Furthermore, many physical systems are sensitive to many unknown disturbances, such as external perturbation and parametric variations, making these approaches challenging to apply practically.

In the sense of robust nonlinear control algorithms, the sliding mode control (SMC) has received much interest [22]. In the presence of disturbances, this strategy will drive the dynamics of UNS to meet the desired target, like the double-pendulum crane in [13], unmanned aerial vehicle (UAV) in [32], cartpendulum system in [21], satellite in [10] and ball and beam system in $[5,1]$. However, the chattering phenomenon has become more prevalent in UNS because of the high dynamics coupling and nonlinear solid terms. To circumvent this shortcoming, various higher-order sliding mode techniques have been used (see for instance $[5,4,2,14]$ ). In controlling UNS dynamics, the control strategies developed in $[5,4]$ produce some very impressive findings. These methods were fascinating, but due to their asymptotic convergence, the steady-state error resulted in less accuracy. To eliminate the chattering problem and get high precision, [12] presented a fast TSMC law. Compared to conventional linear SMC, this method achieves finite-time convergence and delivers excellent robustness with high precision. However, the negative fractional powers in its sliding manifold, this scheme may cause the singularity issue ([30]). There have been significant research efforts on the finite-time control method for applications that need a time response constraint, such as robotic manipulators [16], guidance systems [19], and spacecraft [15]. This technique not only guarantees finite-time enforcement of the system states to the origin, but it 
also assures high precision [17]. However, its disadvantage is that initial conditions require increased values for the kinematic state components, leading to a longer convergence duration [24]. As a result, an accurate prior estimate of the settling time can not be made [33]. As the finite time system states converge, this scheme is closely related to the states' initial condition; therefore, this law can not be used in realistic application if the prior initial state conditions are uncertain. However, the finite-time TSMC's convergence speed is determined by the values of initial system states, which leads to a longer stabilization time when the values of the initial system state are high.

The literature [20] developed fixed-time convergence theory, an extension of the finite-time convergence theory, to tackle this problem. In comparison to the finite-time stability, the fixed-time stability possessed optimal stability within bounded time without knowing the initial conditions. This algorithm has been widely used in analysis over the last two decades due to its outstanding characteristics. A fast fixed-time non-singular TSMC law was introduced in [18] to compensate for the chaos in power systems. The control researchers in [11] proposed a new non-singular fixed-time fast TSMC scheme for the control of second-order multi-agent systems. The literature [29] developed a novel fixed-time non-singular TSMC approach to control maneuvering objectives. A fixed-time robust non-singular TSMC law was proposed in the literature [3] for the control of uncertain nonlinear systems. For the stability of a single cart-pendulum system, another non-singular fixed-time TSMC technique was introduced in [25]. Nevertheless, these approaches were either limited in their applicability or had theoretical problems such as chattering, step life, and poor robustness in the entire operation of system dynamics [7]. Several neural networks are used in the controller architecture to increase robustness by estimating the external disturbances and uncertain nonlinearities (see [23] and references therein).

In this paper, a new non-singular fast TSMC strategy is proposed for the fixed-time control of uncertain under-actuated nonlinear systems and eliminating the associated singularity problem associated with the classical TSMC scheme. The main contributions of this work are three-fold. The first one is transforming the generalized dynamical model of the understudy class of $n$ degree of freedom into its equivalent control convenient regular form. The transformed regular form divides the overall dynamical model into two blocks named directly driven block and indirectly driven block by the applied control actuator. The second contribution is the employment of RBF-based neural networks to estimate the highly nonlinear drift functions, which can suppress the strong influence of uncertain disturbances. The last contribution is developing a fixed-time TSMC framework for the transformed regular form to ensure significant control characteristics with fast convergence and high precision. The proposed control synthesis annihilates the singularity issue and ensures minimum settling time for any initial state conditions. The step-by-step proof of fixed-time stabilization of both system states and sliding manifolds is presented through the Lyapunov stability function. The theoretical analysis and MATLAB simulations of two benchmark examples (cart-pendulum and quadcopter 
systems) demonstrate the feasibility and benefits of the non-singular coordinate transformation, the proposed control synthesis, and RBF-based functions estimator. The simulations of these benchmarks are compared with the standard literature to show the proposed control scheme's attractive nature for the under-study class. This paper proceeds as follows: The dynamical model description of the under-study problem and its regular form transformation are introduced in Section 2. In Section 3, the proposed control technique's design procedure is given to control the under-study class. Two benchmark examples of cart-pendulum and quadcopter, along with detailed discussions of simulation results, are demonstrated in Section 4 . The concluding remarks are summarized in the last section.

\section{Problem Formulation}

Consider the following motion equation for any mechanical system in vector form

$$
\mathfrak{J}(q) \ddot{q}+\mathfrak{F}_{b}(\dot{q})+\mathfrak{F}_{g}(q)+\mathfrak{F}_{c}(q, \dot{q}) \dot{q}=\mathfrak{F}_{e}(q) U
$$

where $q, \dot{q} \in R^{n}$ and $U \in R$ are the system states and applied control input, respectively, $\mathfrak{F}_{b}(\dot{q})$ are the fractional forces, $\mathfrak{F}_{g}(q)$ are gravitational forces and $\mathfrak{F}_{c}(q, \dot{q})$ points to coriolis force/ or centrifugal force (see [26] for more details). The system in equation (1) can be written in the following family of $\mathrm{n}$ underactuated systems.

$$
\left.\begin{array}{c}
\ddot{q}_{1}=F_{1}(q, \dot{q})+G_{1}(q) U \\
\ddot{q}_{2}=F_{2}(q, \dot{q})+G_{2}(q) U \\
\vdots \\
\ddot{q}_{n}=F_{n}(q, \dot{q})+G_{n}(q) U
\end{array}\right\}
$$

Note that a signal control input $U$ is used to control all configuration variables. For simplicity and without loss of generality, (2) can be presented in the following comprehensive style.

$$
\left.\begin{array}{rl}
\ddot{q}_{i} & =F_{i}(q, \dot{q})+G_{i}(q) U \\
\ddot{q}_{n} & =F_{n}(q, \dot{q})+G_{n}(q) U
\end{array}\right\} i=1,2, \ldots, n-1
$$

Assumption 1 According to the controllability condition, the drift function $G_{n}$ is assumed to be a function of the non-zero value for $q$ and $t$ [27].

Remark 1 Since the dynamical model (3) is coupled both in inputs and states. Therefore, the following non-singular coordinate transformation are presented to reduce the dynamical model into a control convenient regular form.

$$
y_{i}=q_{i}-\psi_{i}\left(q_{n}, t\right) \quad \text { and } \quad z_{n}=q_{n}
$$


where $q_{i}=\psi_{i}\left(q_{n}, t\right)$ is the solution of $\frac{d q_{i}}{d q_{n}}=\frac{\partial}{\partial q_{n}} \psi_{i}\left(q_{n}, t\right) \dot{q}_{n}=\frac{G_{i}}{G_{n}}$. Consequently, the following regular form is achieved.

$$
\left.\begin{array}{rl}
\dot{y}_{i} & =\dot{q}_{i}-\frac{\partial}{\partial q_{n}} \psi_{i}\left(q_{n}, t\right) \dot{q}_{n}=F_{i}-\frac{G_{i}}{G_{n}} F_{n} \\
\dot{z}_{n} & =\dot{q}_{n} \\
\ddot{y}_{i} & =\bar{F}_{i}\left(y_{i}, \dot{y}_{i}, z_{n}, \dot{z}_{n}\right) \\
\ddot{z}_{n} & =\bar{F}_{n}\left(y_{i}, \dot{y}_{i}, z_{n}, \dot{z}_{n}\right)+\bar{G}_{n}\left(y_{i}, z_{n}\right) U
\end{array}\right\}
$$

The transformed dynamic model (4) can be expressed in the following state space form.

$$
\left\{\begin{array}{l}
\dot{y}_{i 1}=y_{i 2} \\
\dot{y}_{i 2}=\bar{F}_{i}\left(y_{i 1}, z_{n 1}, y_{i 2}, z_{n 2}\right) \\
\dot{z}_{n 1}=z_{n 2} \\
\dot{z}_{n 2}=\bar{F}_{n}\left(y_{i 1}, z_{n 1}, y_{i 2}, z_{n 2}\right)+\bar{G}_{n}\left(y_{i 1}, z_{n 1}\right) U+\Delta_{n}\left(y_{i 1}, z_{n 1}, t\right)
\end{array}\right.
$$

where $y_{i 1}=y_{i}, y_{i 2}=\dot{y}_{i}, z_{n 1}=z_{n}$ and $z_{n 2}=\dot{z}_{n}$. The $y_{i}$ dynamics are known as the internal dynamics (which are not directly depend on the applied control input $U$ ) and the $z_{n}$ dynamics are named as the visible dynamics (that are directly controlled by $U$ ). The term $\Delta\left(y_{i 1}, z_{n 1}, t\right)$ (uncertainty of matched/unmatched nature) is assumed to be less or equal to a positive constant.

Remark 2 The main objective of this work is to investigate a fixed-time control algorithm that will track the $y$-dynamics on the desired trajectories while regulating the $z$-dynamics to the equilibrium points.

The step-by-step design procedure of the proposed method for the proposed control problem will be investigated in the next section.

\section{Control Law Design}

In this section, the step by step control design of RBF neural network estimator based non-singular TSMC synthesis is presented to enforce both the internal dynamics $y_{i}$ and visible dynamics $z_{n}$ dynamics of the considered class of underactuated electromechanical systems to their equilibrium points in fixed-time.

\subsection{Fixed-time TSM control law design}

For this purpose, the following error variable is presented to track the actual internal dynamics at its desired trajectory $y_{i}^{\star}$.

$$
\xi_{i}=y_{i 1}-y_{i}^{\star}
$$


The time derivatives of the error variables $\xi_{i}$ can be evaluated as follow

$$
\begin{aligned}
& \dot{\xi}_{i}=y_{i 2}-\dot{y}_{i}^{\star} \\
& \ddot{\xi}_{i}=\bar{F}_{i}\left(y_{i 1}, z_{n 1}, y_{i 2}, z_{n 2}\right)-\ddot{y}_{i}^{\star} \\
& \dddot{\xi}_{i}=\frac{\partial \bar{F}_{i}}{\partial y_{i 1}} \dot{y}_{i 1}+\frac{\partial \bar{F}_{i}}{\partial z_{n 1}} \dot{z}_{1}+\frac{\partial \bar{F}_{i}}{\partial y_{i 2}} \dot{y}_{i 2}+\frac{\partial \bar{F}_{i}}{\partial z_{n 2}} \dot{z}_{2}-\dddot{y}_{i}^{\star}
\end{aligned}
$$

The fixed-time convergence of $y_{i}$ dynamics can be guaranteed by defining the following non-singular fast terminal sliding manifold.

$$
\$_{i}=\dot{\xi}_{i}+\alpha_{i}\left|\xi_{i}\right|^{\kappa_{i}} \operatorname{sign}\left(\xi_{i}\right)+\beta_{i} \chi_{i}\left(\xi_{i}\right)
$$

with

$$
\chi_{i}\left(\xi_{i}\right)= \begin{cases}\left|\xi_{i}\right|^{\gamma_{i}} \operatorname{sign}\left(\xi_{i}\right), & \left|\xi_{i}\right| \geq \rho_{i} \\ \bar{\chi}_{i} \sin \left(\xi_{i}\right)+\varsigma_{i}\left|\xi_{i}\right|^{\epsilon_{i}} \operatorname{sign}\left(\xi_{i}\right), & \left|\xi_{i}\right|<\rho_{i}\end{cases}
$$

where $\alpha_{i}>0, \beta_{i}>0 \kappa_{i}=0.5\left(\mu_{i}+1\right)+0.5\left(\mu_{i}-1\right) \operatorname{sign}\left(\left|\xi_{i}\right|-1\right), \gamma_{i}=$ $0.5\left(\mu_{i}+\eta_{i}\right)+0.5\left(\mu_{i}-\eta_{i}\right) \operatorname{sign}\left(\left|\xi_{i}\right|-1\right), \mu>1, \bar{\chi}_{i}=\frac{\eta_{i} \varrho_{i}^{\eta_{i}}-\epsilon_{i} \varrho_{i}^{\eta_{i}}}{\varrho_{i} \cos \left(\varrho_{i}\right)-\epsilon_{i} \sin \left(\varrho_{i}\right)}, \varsigma_{i}=$ $\frac{\eta_{i} \varrho_{i}^{\eta_{i}-1}-\bar{\chi}_{i} \cos \left(\varrho_{i}\right)}{\epsilon_{i} \varrho_{i}-1}, 0<\rho_{i}<1,0<\eta_{i}<1$ and $1<\epsilon_{i}<2$.

The proposed sliding surface (8) can be expended to following three parts.

$$
\$_{i}= \begin{cases}\dot{\xi}_{i}+\alpha_{i}\left|\xi_{i}\right|^{\mu_{i}} \operatorname{sign}\left(\xi_{i}\right)+\beta_{i}\left|\xi_{i}\right|^{\mu_{i}} \operatorname{sign}\left(\xi_{i}\right), & \left|\xi_{i}\right|>1 \\ \dot{\xi}_{i}+\alpha_{i} \xi_{i}+\beta_{i}\left|\xi_{i}\right|^{\eta_{i}} \operatorname{sign}\left(\xi_{i}\right), & \rho_{i}<\left|\xi_{i}\right| \leq 1 \\ \dot{\xi}_{i}+\alpha_{i} \xi_{i}+\beta_{i}\left(\bar{\chi}_{i}\left|\xi_{i}\right| \operatorname{sign}\left(\xi_{i}\right)+\varsigma_{i}\left|\xi_{i}\right|^{\epsilon_{i}} \operatorname{sign}\left(\xi_{i}\right)\right), & \left|\xi_{i}\right| \leq \rho_{i}\end{cases}
$$

Remark 3 Equation (10) ensures the fast convergence rate either the system states are far from or close to the desired equilibria. In addition, the elimination of singularity issues associated with conventional TSM is also claimed by the proposed sliding surface.

Now, the time differentiation of sliding manifold (9) can be evaluated as

$$
\dot{\$}_{i}=\ddot{\xi}_{i}+\alpha_{i} \kappa_{i}\left|\xi_{i}\right|^{\kappa_{i}-1} \dot{\xi}_{i}+\beta_{i} \dot{\chi}_{i}\left(\xi_{i}\right)
$$

with

$$
\dot{\chi}_{i}\left(\xi_{i}\right)= \begin{cases}\gamma_{i}\left|\xi_{i}\right|^{\gamma_{i}-1} \dot{\xi}_{i}, & \left|\xi_{i}\right| \geq \rho_{i} \\ \bar{\chi}_{i} \cos \left(\xi_{i}\right) \dot{\xi}_{i}+\varsigma_{i} \epsilon_{i}\left|\xi_{i}\right|^{\epsilon_{i}-1} \dot{\xi}_{i}, & \left|\xi_{i}\right|<\rho_{i}\end{cases}
$$

Note that the achievement of $\ddot{\xi}_{i}=-\$_{i}$, in sliding phase, is the primary requirement of this work. Therefore, along the following terminal attractor, the steering of sliding mode is essential to meet this requirement.

$$
\Upsilon_{i}=\ddot{\xi}_{i}+\$_{i}=\bar{F}_{i}\left(y_{i 1}, z_{n 1}, y_{i 2}, z_{n 2}\right)-\ddot{y}_{i}^{\star}+\$_{i}
$$

When the sliding mode is enforced then the forthcoming fixed-time stable second order system will be used to control the zero dynamics of (5). Furthermore, the system states of (5) will achieve the fast convergence in fixed-time, i.e.,

$$
\ddot{\xi}_{i}+\dot{\xi}_{i}+\alpha_{i}\left|\xi_{i}\right|^{\kappa_{i}} \operatorname{sign}\left(\xi_{i}\right)+\beta_{i} \chi_{i}\left(\xi_{i}\right)=0
$$


In this case, the sliding variable $\Upsilon_{i}$ treated as a virtual output in visible dynamics block, and the drift function $\bar{F}_{i}$ is acted as a virtual input to the internal dynamics block in (44).

Remark 4 If the system relative degree is more significant than one (i.e., the nonlinear distribution function $\bar{F}_{i}$ in (5) does not include $\dot{z}$ ), then another fixed-time terminal attractor will be defined to increase its relative degree and consequently, ensure the control of dynamics expressed in (14).

$$
\Upsilon_{n}=\dot{\Upsilon}_{i}+\alpha_{n}\left|\Upsilon_{i}\right|^{\kappa_{n}} \operatorname{sign}\left(\Upsilon_{i}\right)+\beta_{n} \chi_{n}\left(\Upsilon_{i}\right)
$$

with

$$
\chi_{n}\left(\Upsilon_{i}\right)= \begin{cases}\left|\Upsilon_{i}\right|^{\gamma_{n}} \operatorname{sign}\left(\Upsilon_{i}\right), & \left|\Upsilon_{i}\right| \geq \rho_{n} \\ \bar{\chi}_{n} \sin \left(\Upsilon_{i}\right)+\varsigma_{n}\left|\Upsilon_{i}\right|^{\epsilon_{n}} \operatorname{sign}\left(\Upsilon_{i}\right), & \left|\Upsilon_{i}\right|<\rho_{n}\end{cases}
$$

Remark 5 The following reaching law is presented to tackle the associated chattering issue with conventional SMC.

$$
\dot{\Upsilon}_{i}=-\bar{\alpha}_{i}\left|\Upsilon_{i}\right|^{\bar{\kappa}_{i}} \operatorname{sign}\left(\Upsilon_{i}\right)-\bar{\beta}_{i}\left|\Upsilon_{i}\right|^{\bar{\gamma}_{i}} \operatorname{sign}\left(\Upsilon_{i}\right)
$$

where $\bar{\alpha}_{i}, \bar{\kappa}_{i}, \bar{\beta}_{i}$ and $\bar{\gamma}_{i}$ are positive controller gains.

The forthcoming theorem details the fixed-time convergence of the under-study class of UNS.

Theorem 1 Given the dynamical model of UNS as defined in (5) with the fast terminal attractor expressed in (15) and the reachability law selected in (17). Then, the following control scheme ensures the fast convergence of sliding mode, in fixed-time, against the terminal attractor.

$$
\begin{aligned}
U= & \bar{G}_{n}^{-1}\left(\frac { \partial \overline { F } _ { i } } { \partial z _ { n 2 } } \left(-\bar{\alpha}_{i}\left|\Upsilon_{i}\right|^{\bar{\kappa}_{i}} \operatorname{sign}\left(\Upsilon_{i}\right)-\bar{\beta}_{i}\left|\Upsilon_{i}\right|^{\bar{\gamma}_{i}} \operatorname{sign}\left(\Upsilon_{i}\right)-\frac{\partial \bar{F}_{i}}{\partial y_{i 1}} \dot{y}_{i 1}\right.\right. \\
& \left.\left.-\frac{\partial \bar{F}_{i}}{\partial z_{n 1}} \dot{z}_{1}-\frac{\partial \bar{F}_{i}}{\partial y_{i 2}} \dot{y}_{i 2}+\dddot{y}_{i}^{\star}-\$_{i}\right)-\bar{F}_{n}\right)
\end{aligned}
$$

Consequently, the system states will be enforced to their desired equilibria.

Proof: To prove this theorem, differentiate the given terminal attractor in (13) along the dynamics of system (5).

$$
\dot{\Upsilon}_{i}=\frac{\partial \bar{F}_{i}}{\partial y_{i 1}} \dot{y}_{i 1}+\frac{\partial \bar{F}_{i}}{\partial z_{n 1}} \dot{z}_{1}+\frac{\partial \bar{F}_{i}}{\partial y_{i 2}} \dot{y}_{i 2}+\frac{\partial \bar{F}_{i}}{\partial z_{n 2}}\left(\bar{F}_{n}+\bar{G}_{n} U+\Delta\right)-\dddot{y}_{i}^{\star}+\dot{\$}_{i}
$$

The time differentiation of Lyapunov function $\Lambda_{i}=\frac{1}{2} \Upsilon_{i}^{2}$ along (19) can be evaluated as follows

$$
\dot{\Lambda}_{i}=\Upsilon_{i}\left(\frac{\partial \bar{F}_{i}}{\partial y_{i 1}} \dot{y}_{i 1}+\frac{\partial \bar{F}_{i}}{\partial z_{n 1}} \dot{z}_{1}+\frac{\partial \bar{F}_{i}}{\partial y_{i 2}} \dot{y}_{i 2}+\frac{\partial \bar{F}_{i}}{\partial z_{n 2}}\left(\bar{F}_{n}+\bar{G}_{n} U+\Delta\right)-\dddot{y}_{i}^{\star}+\dot{\$}_{i}\right)
$$


Therefore, by substituting (18) in (20), one can get the following expression.

$$
\dot{\Lambda}_{i}=\Upsilon_{i}\left(-\bar{\alpha}_{i}\left|\Upsilon_{i}\right|^{\overline{\mid}_{i}} \operatorname{sign}\left(\Upsilon_{i}\right)-\bar{\beta}_{i}\left|\Upsilon_{i}\right|^{\bar{\gamma}_{i}} \operatorname{sign}\left(\Upsilon_{i}\right)+\frac{\partial \bar{F}_{i}}{\partial z_{n 2}} \Delta\right)
$$

Considering the identity $\left|\Upsilon_{i}\right|=\Upsilon_{i} \operatorname{sign}\left(\Upsilon_{i}\right)$, (21) can be re-written as follows

$$
\dot{\Lambda}_{i} \leq-\bar{\alpha}_{i}\left|\Upsilon_{i}\right|^{\bar{\kappa}_{i}+1}-\left(\bar{\beta}_{i}+|\tilde{\Delta}|\right)\left|\Upsilon_{i}\right|^{\bar{\gamma}_{i}+1} \leq-\bar{\alpha}_{i}\left|\Upsilon_{i}\right|^{\bar{\kappa}_{i}+1}-\tilde{\beta}_{i}\left|\Upsilon_{i}\right|^{\bar{\gamma}_{i}+1}
$$

where $\tilde{\Delta}=\frac{\partial \bar{F}_{i}}{\partial z_{n 2}} \frac{\Delta}{\left|\Upsilon_{i}\right|^{\gamma_{i}}}$. It is worth noting that the above inequality (22) remains true subject to $\tilde{\beta}_{i} \leq \bar{\beta}_{i}+|\tilde{\Delta}|$. It may also be written as

$$
\dot{\Lambda}=-\dot{\alpha}_{i}|\Lambda|^{\frac{\bar{\kappa}_{i}+1}{2}}-\dot{\beta}_{i}|\Lambda|^{\frac{\bar{\gamma}_{i}+1}{2}}
$$

where $\alpha_{i}=\sqrt{2} \bar{\alpha}_{i}$ and $\hat{\beta}_{i}=\sqrt{2} \tilde{\beta}_{i}$.

Thus, the sliding mode establishment, against the fixed-time terminal attractor, has been proved. The following differential equation for the system (17) is presented to find its upper bound of convergence time.

$$
\dot{\Upsilon}_{i}= \begin{cases}-\bar{\alpha}_{i}\left|\Upsilon_{i}\right|^{\bar{\mu}_{i}} \operatorname{sign}\left(\Upsilon_{i}\right)-\bar{\beta}_{i}\left|\Upsilon_{i}\right|^{\bar{\mu}_{i}} \operatorname{sign}\left(\Upsilon_{i}\right), & \left|\Upsilon_{i}\right|>1 \\ -\bar{\alpha}_{i} \Upsilon_{i}-\bar{\beta}_{i}\left|\Upsilon_{i}\right|^{\bar{\eta}_{i}} \operatorname{sign}\left(\Upsilon_{i}\right), & \left|\Upsilon_{i}\right| \leq 1\end{cases}
$$

By solving (24), the upper bound of convergence time can be evaluated as

$$
\begin{aligned}
t_{r}= & \lim _{\Upsilon_{i}(0) \rightarrow \infty}\left(\int_{1}^{\Upsilon_{i}(0)} \frac{1}{\left(\bar{\alpha}_{i}+\bar{\beta}_{i}\right)\left|\Upsilon_{i}\right|^{\bar{\mu}_{i}} \operatorname{sign}\left(\Upsilon_{i}\right)} d \Upsilon_{i}+\int_{0}^{1} \frac{1}{\bar{\alpha}_{i} \Upsilon_{i}+\bar{\beta}_{i}\left|\Upsilon_{i}\right| \bar{\eta}_{i} \operatorname{sign}\left(\Upsilon_{i}\right)} d \Upsilon_{i}\right) \\
& <\frac{1}{\left(\alpha_{i}+\beta_{i}\right)\left(\mu_{i}-1\right)}+\frac{1}{\beta_{i}\left(1-\eta_{i}\right)} \ln \left(1+\frac{\alpha_{i}}{\beta_{i}}\right)
\end{aligned}
$$

Having enforced the sliding mode, the convergence of system states can be analyzed by considering (8).

$$
\$_{i}=\dot{\xi}_{i}+\alpha_{i}\left|\xi_{i}\right|^{\kappa_{i}} \operatorname{sign}\left(\xi_{i}\right)+\beta_{i} \chi_{i}\left(\xi_{i}\right)=0
$$

The time differentiation of the augmented Lyapunov function $\Lambda_{i 1}=\frac{1}{2} \xi_{i}^{2}$ can be calculated as

$$
\begin{aligned}
\dot{\Lambda}_{i 1} & =\xi_{i}\left(-\alpha_{i}\left|\xi_{i}\right|^{\kappa_{i}} \operatorname{sign}\left(\xi_{i}\right)-\beta_{i}\left|\xi_{i}\right|^{\gamma_{i}} \operatorname{sign}\left(\xi_{i}\right)\right)=-\alpha_{i}\left|\xi_{i}\right|^{\kappa_{i}+1}-\beta_{i}\left|\xi_{i}\right|^{\gamma_{i}+1} \\
& =-\dot{\alpha}_{i}\left|\Lambda_{2}\right|^{\frac{\kappa_{i}+1}{2}}-\dot{\beta}_{i}\left|\Lambda_{2}\right|^{\frac{\gamma_{i}+1}{2}}
\end{aligned}
$$

where $\grave{\alpha}_{i}=\sqrt{2} \alpha_{i}$ and $\grave{\beta}_{i}=\sqrt{2} \beta_{i}$. From (27), the convergence time $t_{s}$ of the system states, required to drive to their equilibrium points, is bounded by

$$
t_{s}<\frac{1}{\left(\grave{\alpha}_{i}+\grave{\beta}_{i}\right)\left(\grave{\mu}_{i}-1\right)}+\frac{1}{\grave{\beta}_{i}\left(1-\grave{\eta}_{i}\right)} \ln \left(1+\frac{\grave{\alpha}_{i}}{\grave{\beta}_{i}}\right)
$$

Thus, the proof is completed. In this study, the designed fixed-time control law (18) is used to establish sliding mode against the proposed fixed-time terminal 
attractor (15). Having confirmed sliding mode, $\Upsilon_{i} \longrightarrow 0$ will be satisfied. Consequently, it will confirm the tracking of system outputs on the desired trajectory in fixed-time, i.e., $y_{i} \longrightarrow y_{i}^{\star}$. The total convergence time is the algebraic sum of $t_{s}$ and $t_{r}$.

In the following subsection, some nonlinear drift functions are estimated via an advanced neural network method.

\subsection{Neuro-adaptive fixed-time TSM control law design}

The control input $U$, developed in theorem 1, requires the estimates of the highly nonlinear drift functions in feedback. Therefore, a radial basis functionbased neural network is proposed, in this subsection, to estimate these uncertain functions adaptively. Consequently, it ensures chattering elimination and robust performance. In this algorithm, the network weight matrices are adjusted online according to some adaptive control laws developed via the Lyapunov stability function. Because of these network weights, the estimated functions adapt and ensure the target data at the running condition. Whenever there is a new situation in the system, the network will improve its knowledge to handle this situation. The system states $x=\left[y_{i 1}, y_{i 2}, z_{n 1}, z_{n 2}\right] \in \mathbb{R}^{n}$ are the network input vector and the estimated functions $\hat{F}_{n}, \hat{G}_{n}$ are the network targets. The network output is expressed as follows

$$
\begin{aligned}
\hat{F}_{n} & =\hat{W}_{j_{F}} h_{j}(x) \\
\hat{G}_{n} & =\hat{W}_{j_{G}} h_{j}(x)
\end{aligned}
$$

where $\hat{W}_{j}=\left[\hat{W}_{1}, \hat{W}_{2}, \ldots, \hat{W}_{l}\right]^{T}$ is the network weight matrix and $h_{j}(x)=$ $\left[h_{1}(x), h_{2}(x), \ldots, h_{l}(x)\right]^{T}$ is the Gaussian function vector, which is introduced as follows

$$
h_{j}(x)=\exp \left(-\frac{\left(x-c_{j k}\right)^{T}\left(x-c_{j k}\right)}{2 b_{j}^{2}}\right)
$$

where $b_{j}$ is the width of Gaussian function, $k$ is the network input number, $c_{j k}=\left[c_{j 1}, c_{j 2}, \ldots, c_{j q}\right]^{T}$ is the center vector and $j$ is the number of hidden layer network nodes. For any $e>0$, the following inequality holds.

$$
\begin{aligned}
\bar{F}_{n} & =W_{j_{F}} h_{j}(x)+e_{F}(x) \\
\bar{G}_{n} & =W_{j_{G}} h_{j}(x)+e_{G}(x)
\end{aligned}
$$

where

$$
\begin{aligned}
& \tilde{F}_{n}=\hat{F}_{n}-\bar{F}_{n}=\tilde{W}_{j_{F}} h_{j}(x)-e_{F}(x) \\
& \tilde{G}_{n}=\hat{G}_{n}-\bar{G}_{n}=\tilde{W}_{j_{G}} h_{j}(x)-e_{G}(x) \\
& \tilde{W}_{j_{F}}=\hat{W}_{j_{F}}-W_{j_{F}}, \quad \dot{\tilde{W}}_{j_{F}}=\dot{\hat{W}}_{j_{F}} \\
& \tilde{W}_{j_{G}}=\hat{W}_{j_{G}}-W_{j_{G}}, \quad \dot{\tilde{W}}_{j_{G}}=\dot{\hat{W}}_{j_{G}}
\end{aligned}
$$


and $e_{F}, e_{G}$ are network approximation errors which are bounded over a compact set, i.e., $|e(x)| \leq \xi$ with $\xi>0$ an unknown constant.

Having invoked the proposed network, (18) can be re-designed in the following form.

$$
\begin{aligned}
U= & \hat{G}_{n}^{-1}\left(-\hat{F}_{n}-\frac{\partial \bar{F}_{i}}{\partial z_{n 2}}\left(-\bar{\alpha}_{i}\left|\Upsilon_{i}\right|^{\bar{\kappa}_{i}} \operatorname{sign}\left(\Upsilon_{i}\right)-\bar{\beta}_{i}\left|\Upsilon_{i}\right|^{\bar{\gamma}_{i}} \operatorname{sign}\left(\Upsilon_{i}\right)\right.\right. \\
& +\frac{\partial \bar{F}_{i}}{\partial y_{i 1}} \dot{y}_{i 1}+\frac{\partial \bar{F}_{i}}{\partial z_{n 1}} \dot{z}_{1}+\frac{\partial \bar{F}_{i}}{\partial y_{i 2}} \dot{y}_{i 2}+\ddot{\xi}_{i}+\alpha_{i} \kappa_{i}\left|\xi_{i}\right|^{\kappa_{i}-1} \dot{\xi}_{i}-\dddot{y}_{i d} \\
& \left.\left.+\beta_{i} \dot{\chi}_{i}\left(\xi_{i}\right)+\alpha_{n} \kappa_{n}\left|\Upsilon_{i}\right|^{\kappa_{n}-1} \dot{\Upsilon}_{i}+\beta_{n} \dot{\chi}_{n}\left(\Upsilon_{i}\right)\right)\right)
\end{aligned}
$$

Theorem 2 Consider the dynamical model of under-study class (5) along with the fixed-time terminal sliding manifold (15) and the reachability law (17). Then, the neuro-adaptive control input developed in (33) guarantees the establishment of sliding mode in fixed-time.

Proof:The following Lyapunov function is presented to approximate the network weights.

$$
\Lambda=\frac{1}{2} \Upsilon_{i}^{2}+\frac{1}{2 \Upsilon_{1}} \tilde{W}_{j_{F}}^{T} \tilde{W}_{j_{F}}+\frac{1}{2 \Upsilon_{2}} \tilde{W}_{j_{G}}^{T} \tilde{W}_{j_{G}}
$$

The time differentiation of (34) along (19) can be evaluated as follows

$$
\begin{aligned}
\dot{\Lambda}= & \Upsilon_{i}\left(\frac{\partial \bar{F}_{i}}{\partial y_{i 1}} \dot{y}_{i 1}+\frac{\partial \bar{F}_{i}}{\partial z_{n 1}} \dot{z}_{1}+\frac{\partial \bar{F}_{i}}{\partial y_{i 2}} \dot{y}_{i 2}+\frac{\partial \bar{F}_{i}}{\partial z_{n 2}}\left(\bar{F}_{n}+\hat{G}_{n} U\right)\right. \\
& -\frac{\partial \bar{F}_{i}}{\partial z_{n 2}}\left(\tilde{G}_{n} U+\Delta\right)+\ddot{\xi}_{i}+\alpha_{i} \kappa_{i}\left|\xi_{i}\right|^{\kappa_{i}-1} \dot{\xi}_{i}-\dddot{y}_{i d}+\beta_{i} \dot{\chi}_{i}\left(\xi_{i}\right) \\
& \left.+\alpha_{n} \kappa_{n}\left|\Upsilon_{i}\right|^{\kappa_{n}-1} \dot{\Upsilon}_{i}+\beta_{n} \dot{\chi}_{n}\left(\Upsilon_{i}\right)\right)+\frac{1}{\Upsilon_{1}} \tilde{W}_{F_{n}}^{T} \dot{\hat{W}}_{F_{n}}+\frac{1}{\Upsilon_{2}} \tilde{W}_{G_{n}}^{T} \dot{\hat{W}}_{G_{n}}
\end{aligned}
$$

Invoking the right side of $U(t)$, one can get

$$
\begin{aligned}
\dot{\Lambda}= & \Upsilon_{i}\left(-\frac{\partial \bar{F}_{i}}{\partial z_{n 2}} \tilde{F}_{n}-\frac{\partial \bar{F}_{i}}{\partial z_{n 2}}\left(\tilde{G}_{n} U+\Delta\right)-\bar{\alpha}_{i}\left|\Upsilon_{i}\right|^{\bar{\kappa}_{i}} \operatorname{sign}\left(\Upsilon_{i}\right)\right. \\
& \left.-\bar{\beta}_{i}\left|\Upsilon_{i}\right|^{\bar{\gamma}_{i}} \operatorname{sign}\left(\Upsilon_{i}\right)\right)+\frac{1}{\Upsilon_{1}} \tilde{W}_{F_{n}}^{T} \dot{\hat{W}}_{F_{n}}+\frac{1}{\Upsilon_{2}} \tilde{W}_{G_{n}}^{T} \dot{\hat{W}}_{G_{n}}
\end{aligned}
$$

Taking (31) and (32) into account, (36) becomes

$$
\begin{aligned}
\dot{\Lambda}= & \Upsilon_{i}\left(\frac{\partial \bar{F}_{i}}{\partial z_{n 2}} \Delta-\frac{\partial \bar{F}_{i}}{\partial z_{n 2}}\left(\tilde{W}_{j_{F}} h_{j}(x)\right)+\frac{\partial \bar{F}_{i}}{\partial z_{n 2}}\left(e_{F}(x)\right)\right. \\
& -\frac{\partial \bar{F}_{i}}{\partial z_{n 2}}\left(\tilde{W}_{j_{G}} h_{j}(x)\right) U+\frac{\partial \bar{F}_{i}}{\partial z_{n 2}}\left(e_{G}(x) U\right)-\bar{\alpha}_{i}\left|\Upsilon_{i}\right|^{\bar{\kappa}_{i}} \operatorname{sign}\left(\Upsilon_{i}\right) \\
& \left.-\bar{\beta}_{i}\left|\Upsilon_{i}\right|^{\bar{\gamma}_{i}} \operatorname{sign}\left(\Upsilon_{i}\right)\right)+\frac{1}{\Upsilon_{1}} \tilde{W}_{F_{n}}^{T} \dot{\hat{W}}_{F_{n}}+\frac{1}{\Upsilon_{2}} \tilde{W}_{G_{n}}^{T} \dot{\hat{W}}_{G_{n}}
\end{aligned}
$$


After considering (29) and (31), one can get

$$
\begin{aligned}
\dot{\Lambda}= & -\bar{\alpha}_{i}\left|\Upsilon_{i}\right|^{\bar{\kappa}_{i}+1}-\bar{\beta}_{i}\left|\Upsilon_{i}\right|^{\bar{\gamma}_{i}+1}+\Upsilon_{i} \frac{\partial \bar{F}_{i}}{\partial z_{n 2}}\left(e_{F}(x)+e_{G}(x) U+\Delta\right) \\
& -\Upsilon_{i}\left(\frac{\partial \bar{F}_{i}}{\partial z_{n 2}}\left(\tilde{W}_{j_{F}} h_{j}(x)\right)\right)-\Upsilon_{i}\left(\frac{\partial \bar{F}_{i}}{\partial z_{n 2}}\left(\tilde{W}_{j_{G}} h_{j}(x)\right) U\right) \\
& +\frac{1}{\Upsilon_{1}} \tilde{W}_{F_{n}}^{T} \dot{\hat{W}}_{F_{n}}+\frac{1}{\Upsilon_{2}} \tilde{W}_{G_{n}}^{T} \dot{\hat{W}}_{G_{n}}
\end{aligned}
$$

From the above (38), the estimated weights are chosen as

$$
\begin{aligned}
\dot{\hat{W}}_{F_{n}} & =\Upsilon_{i} \Upsilon_{1}\left(\frac{\partial \bar{F}_{i}}{\partial z_{n 2}}\left(h_{j}(x)\right)\right) \\
\dot{\hat{W}}_{G_{n}} & =\Upsilon_{i} \Upsilon_{2}\left(\frac{\partial \bar{F}_{i}}{\partial z_{n 2}}\left(h_{j}(x)\right) U\right)
\end{aligned}
$$

The elements of $\frac{\partial \bar{F}_{i}}{\partial z_{n 2}}\left(e_{F}(x)+e_{G}(x) U+\Delta\right)$ are assumed to be norm bounded by a positive constant $L_{c}$, i.e., $\frac{\partial \bar{F}_{i}}{\partial z_{n 2}}\left(e_{F}(x)+e_{G}(x) U+\Delta\right)=L \in \mathbb{R}^{n}$ and $|L|<L_{c}$. Thus

$$
\begin{aligned}
\dot{\Lambda} & =-\bar{\alpha}_{i}\left|\Upsilon_{i}\right|^{\bar{\kappa}_{i}+1}-\bar{\beta}_{i}\left|\Upsilon_{i}\right|^{\bar{\gamma}_{i}+1}+\Upsilon_{i} L \\
& \leq-\bar{\alpha}_{i}\left|\Upsilon_{i}\right|^{\bar{\kappa}_{i}+1}-\left(\bar{\beta}_{i}\left|\Upsilon_{i}\right|^{\bar{\gamma}_{i}}-|L|\right)\left|\Upsilon_{i}\right|
\end{aligned}
$$

Now, if the controller gain $\bar{\beta}_{i}$ is selected such that $\bar{\beta}_{i}\left|\Upsilon_{i}\right|^{\bar{\gamma}_{i}}>|L|$ then (40) can be written as

$$
\dot{\Lambda} \leq-\bar{\alpha}_{i}\left|\Upsilon_{i}\right|^{\bar{\kappa}_{i}+1}-\bar{\beta}_{i}\left|\Upsilon_{i}\right|^{\bar{\gamma}_{i}+1}
$$

The approximation errors $e_{F}$, and $e_{H}$ of the proposed neural network possess a minimal value. Therefore, it will ensure the fast steering of system state $z$ to the equilibrium point in fixed-time.

In the next section, the feasibility of the proposed synthesis is verified with the help of two illustrative examples in the MATLAB environment.

\section{Benchmark Examples}

The brief introduction of the two highly nonlinear under-actuated systems (cart-pendulum and quadcopter UAV) as benchmark examples and their fixedtime TSM control laws are designed and simulated in this section.

\subsection{Cart-pendulum system}

The cart-pendulum system (shown in figure 1) is presented as a benchmark example of under-study class of UNS, which have two configuration variables needed to control by only one control input. The pendulum's pole freely moves around the pivot point, while the cart can only travel in the horizontal plane. 


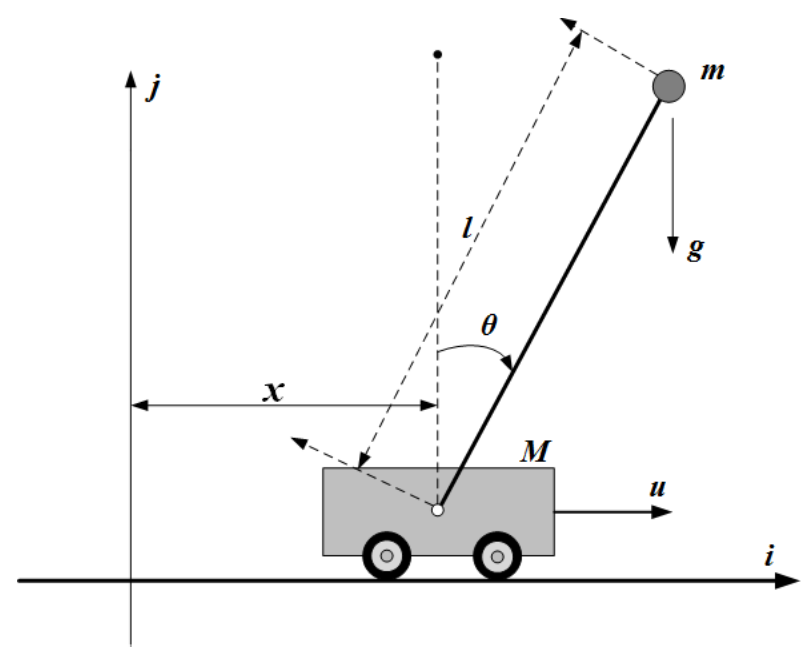

Fig. 1 Cart-pendulum system

\subsubsection{Dynamical model description}

Consider the following uncertain dynamics of the aforesaid system.

$$
\left\{\begin{array}{l}
\ddot{x}=\frac{-1}{\varrho(\theta)}\left(m g \cos \theta \sin \theta-\frac{4}{3} \mathcal{U}\right) \\
\ddot{\theta}=\frac{1}{l \varrho(\theta)}((M+m) g \sin \theta-\cos \theta \mathcal{U})
\end{array}\right.
$$

where $\mathcal{U}=u+m l \dot{\theta}^{2} \sin \theta, \varrho(\theta)=\frac{4}{3}(M+m)-m \cos ^{2} \theta$. The parameter values of cart-pendulum system are rod length $(l)=0.36 \mathrm{~m}$, cart mass $(M)=2.5 \mathrm{~kg}$, gravitational acceleration $g=9.81 \mathrm{~m} / \mathrm{s}, \operatorname{rod} \operatorname{mass}(\mathrm{m})=0.23 \mathrm{~kg}$ and the moment of inertia $J=0.099 \mathrm{~kg} \cdot \mathrm{m}^{2}$. By introducing the following non-singular coordinate,

$$
\dot{y}=\dot{x}+\frac{4 l}{3 \cos \theta} \dot{\theta} \Rightarrow \ddot{y}=\ddot{x}+\frac{4 l \sin \theta}{3 \cos ^{2} \theta} \dot{\theta}^{2}+\frac{4 l}{3 \cos \theta} \ddot{\theta}
$$

and invoking $\ddot{x}$ and $\ddot{\theta}$ given in (42), the dynamical system becomes

$$
\begin{cases}\dot{y}_{1}=y_{2}, & \dot{y}_{2}=\bar{F}_{1}\left(\theta_{1}, \theta_{2}\right) \tan \theta_{1} \\ \dot{\theta}_{1}=\theta_{2}, & \dot{\theta}_{2}=\bar{F}_{2}\left(\theta_{1}\right)+\bar{G}_{2}\left(\theta_{1}\right) U\end{cases}
$$

where $\bar{F}_{1}=\frac{g}{\varrho}\left(\left(\frac{4}{3}-\cos ^{2} \theta_{1}\right) m+\frac{4}{3} M\right)+\frac{4}{3} \frac{l \theta_{2}^{2}}{\cos \theta_{1}}, \bar{F}_{2}=\frac{(M+m) g \sin \theta_{1}}{l \varrho}$ and $\bar{G}_{2}=$ $-\frac{\cos \theta_{1}}{l \varrho}$.

Now, the transformed model (44) is ready for the proposed control design, which is presented in the next subsection. 


\subsubsection{Control design}

Now, the control design $U$ will be designed to enforce the pendulum's pole at a vertically upright unstable position $\left(\theta=0^{\circ}\right)$ while steering the translational cart position at the origin. This law can be developed by defining the mismatch between the cart actual and reference position.

$$
\xi_{1}=y_{1}-y_{d}
$$

The time differentiation of $\xi_{1}$ is evaluated as follows

$$
\left\{\begin{array}{l}
\dot{\xi}_{1}=y_{2}-\dot{y}_{d} \\
\ddot{\xi}_{1}=\tilde{F}_{1}\left(\theta_{1}, \theta_{2}\right) \tan \theta-\ddot{y}_{d} \\
\dddot{\xi}_{1}=\bar{F}_{11}(\theta, \dot{\theta}) \ddot{\theta}+\bar{F}_{12}(\theta, \dot{\theta}) \dot{\theta}-\dddot{y}_{d}
\end{array}\right.
$$

where $\bar{F}_{11}(\theta, \dot{\theta})=\frac{4}{3} \frac{2 l \theta_{2}}{\cos \theta} \tan \theta$ and $\bar{F}_{12}(\theta, \dot{\theta})=\frac{\partial}{\partial \theta}\left(\bar{F}_{1}(\theta, \dot{\theta})\right) \tan \theta+\bar{F}_{1}(\theta, \dot{\theta}) \sec ^{2} \theta$ with $\frac{\partial}{\partial \theta}\left(\bar{F}_{1}(\theta, \dot{\theta})\right)=\left(\frac{2}{\varrho^{2}} m g \cos \theta \sin \theta\left(\left(\left(\frac{4}{3}-\cos ^{2} \theta\right) m+\frac{4}{3} M\right)-\varrho\right)-\frac{4}{3} \frac{l \theta_{2}^{2} \sin \theta}{\cos ^{2} \theta}\right)$. The fast stabilization of $y$ dynamics can be ensured by defining the following sliding surface.

$$
\$_{1}=\dot{\xi}_{1}+\alpha_{1}\left|\xi_{1}\right|^{\kappa_{1}} \operatorname{sign}\left(\xi_{1}\right)+\beta_{1} \chi_{1}\left(\xi_{1}\right)
$$

As, the nonlinear term $\bar{F}_{1}$ has a positive value for all $-\pi / 2 \leq \theta \leq \pi / 2$; therefore, the virtual input $\tan \theta$ is used to control the internal dynamics. The following combination is offered to get this intermediate control.

$$
\tan \theta-\ddot{y}_{d}=-\$_{1}
$$

This can be accomplished by assuming the sliding attractor of the following form.

$$
\Upsilon_{1}=\tan \theta-\ddot{y}_{d}+\$_{1} \Rightarrow \dot{\Upsilon}_{1}=\sec ^{2} \theta \dot{\theta}-\dddot{y}_{d}+\dot{\$}_{1}
$$

Since control input $U$ does not appear in (49), therefore, another terminal attractor is presented as follows

$$
\Upsilon_{2}=\dot{\Upsilon}_{1}+\alpha_{2}\left|\Upsilon_{1}\right|^{\kappa_{2}} \operatorname{sign}\left(\Upsilon_{1}\right)+\beta_{2} \chi_{2}\left(\Upsilon_{1}\right)
$$

The reachability law appears as follows

$$
\dot{\Upsilon}_{2}=-\bar{\alpha}_{2}\left|\Upsilon_{2}\right|^{\bar{\kappa}_{2}} \operatorname{sign}\left(\Upsilon_{2}\right)-\bar{\beta}_{2}\left|\Upsilon_{2}\right|^{\bar{\gamma}_{2}} \operatorname{sign}\left(\Upsilon_{2}\right)
$$

where $\bar{\alpha}_{2}, \bar{\beta}_{2}, \bar{\kappa}_{2}=0.5\left(\bar{\mu}_{2}+1\right)+0.5\left(\bar{\mu}_{2}-1\right) \operatorname{sign}\left(\left|\Upsilon_{2}\right|-1\right), \bar{\gamma}_{2}=0.5\left(\bar{\mu}_{2}+\eta_{2}\right)+$ $0.5\left(\bar{\mu}_{2}-\bar{\eta}_{2}\right) \operatorname{sign}\left(\left|\Upsilon_{2}\right|-1\right), \bar{\mu}_{2}>1$ and $0<\bar{\eta}_{2}<1$ are positive constants.

The final control input can be developed from (50) and (51) as follows

$$
\begin{aligned}
U= & \frac{1}{\bar{G}_{2}\left(\theta_{1}\right)}\left(\frac { 1 } { \operatorname { s e c } ^ { 2 } \theta } \left(-2 \sec ^{2} \theta \tan \theta \dot{\theta}+\dddot{y}_{d}-\ddot{\S}_{1}-\alpha_{2} \kappa_{2}\left|\Upsilon_{1}\right|^{\kappa_{2}-1} \dot{\Upsilon}_{1}\right.\right. \\
& \left.\left.-\beta_{2} \dot{\chi}_{2}\left(\Upsilon_{1}\right)-\bar{\alpha}_{2}\left|\Upsilon_{2}\right|^{\bar{\kappa}_{2}} \operatorname{sign}\left(\Upsilon_{2}\right)-\bar{\beta}_{2}\left|\Upsilon_{2}\right|^{\bar{\gamma}_{2}} \operatorname{sign}\left(\Upsilon_{2}\right)\right)-\bar{F}_{2}\left(\theta_{1}\right)\right)
\end{aligned}
$$

Consequently, this will drive the system states to the equilibrium points in fixed-time. 


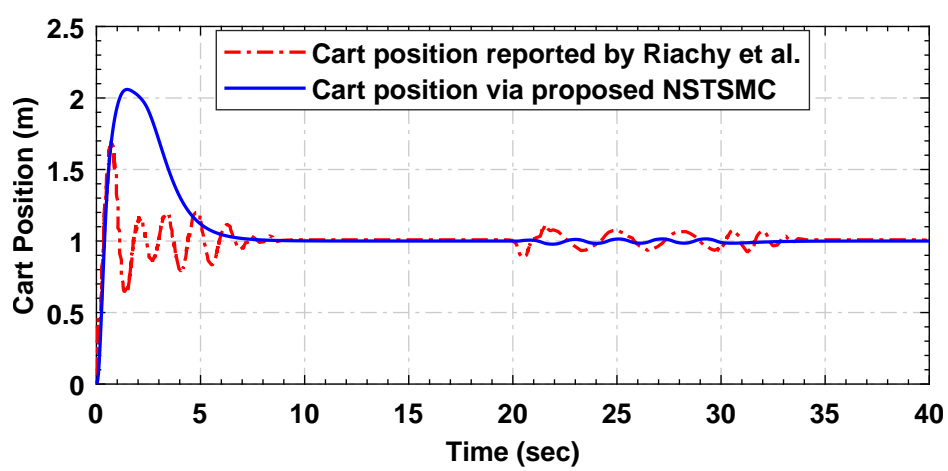

Fig. 2 The cart position tracking performance via the proposed control algorithm in comparison with standard literature [21]

\subsubsection{Result discussion}

Now, the designed control law $U(52)$ is simulated in MATLAB/Simulink environment to verify the applicability of the developed control algorithm.

Figure 2 shows the simulation outcome for the cart location $y_{1}$. As compared to the results of [21], the proposed control method stabilizes the cart position at equilibrium in a short time. Figure 3 depicts the pendulum angle $\theta_{1}$ convergence to its target value. It is apparent from the figure that as compared to the standard result of [21], the pendulum angle is quickly stabilized at the origin via the proposed control technique and then stays there without any oscillation. In figure 4, the regulated input is shown. It's worth mentioning that the control feedback has a virtually chatter-free structure when executing the primary tasks. This technique achieves excellent stabilization with reduced steady-state error and improved robustness in each stage of the control design. The overall calculations were carried out in the presence of matched uncertainties to verify the robustness of the proposed control scheme. Furthermore, some unmatched complexities were inserted into the system, which is tackled in each stage through the virtual control input framework. It's worth mentioning that the newly designed control law is free from high-frequency chattering, which is highly risky in realistic implementations. In other words, the proposed algorithm has significantly decreased the unwanted chattering effects.

\subsection{Quadcopter UAV}

Now, a fixed-time control law is developed for the under-actuated quadcopter system with the goal of achieving complete flight trajectory control, which is further subdivided into two subsystems: fully-actuated and under-actuated. 


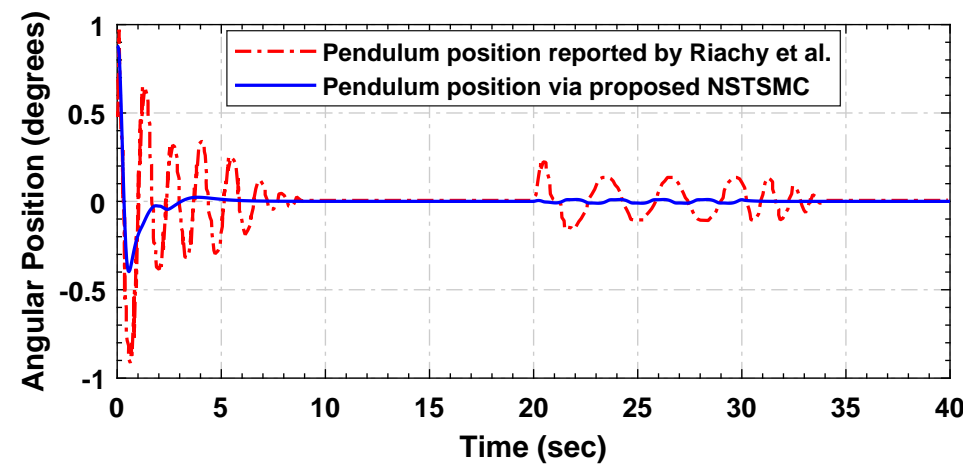

Fig. 3 The rod angle stabilization performance via the proposed control algorithm in comparison with the standard literature [21]

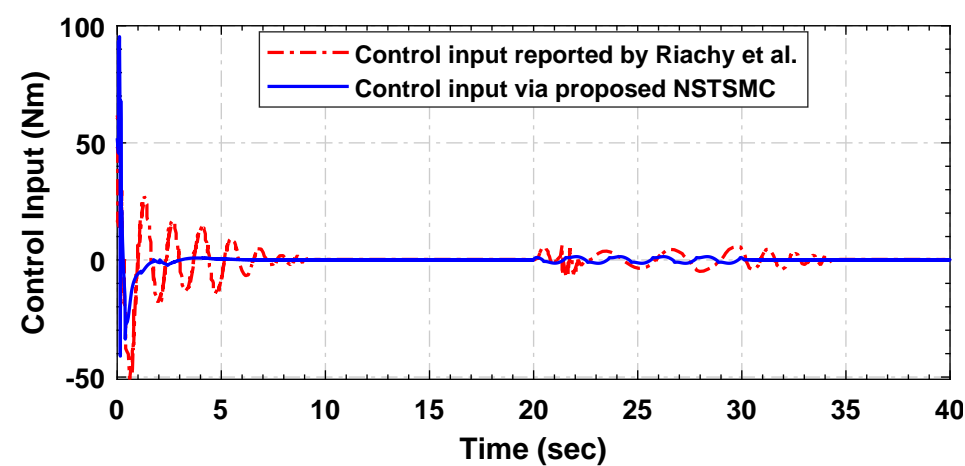

Fig. 4 The control input history of the proposed control algorithm in comparison with the standard literature [21]

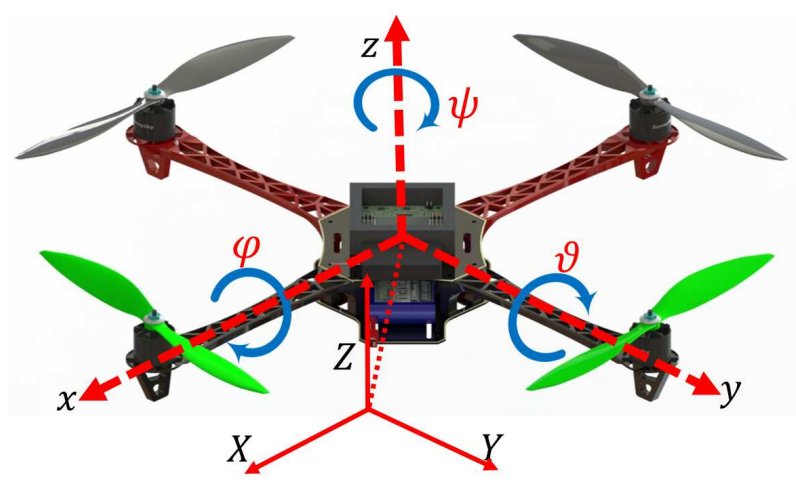

Fig. 5 Quadcopter UAV 


\subsubsection{Dynamical model description}

The quadcopter system (shown in figure figure 5) is typically developed via either Euler-Newton or Euler-Lagrange equations of motion. The following dynamical model of the aforesaid system, having twelve (12) configuration variables, is adopted from [26].

$$
\left.\begin{array}{l}
\ddot{x}=b_{m} £_{x} U_{1} \\
\ddot{y}=b_{m} £_{y} U_{1} \\
\ddot{z}=b_{m} £_{z} U_{1}-g \\
\ddot{\varphi}=J_{x_{1}} \dot{\vartheta} \bar{\mho}+J_{x_{2}} \dot{\vartheta} \dot{\psi}+J_{x_{3}} U_{2} \\
\ddot{\vartheta}=J_{y_{1}} \dot{\varphi} \bar{\mho}+J_{y_{2}} \dot{\varphi} \dot{\psi}+J_{y_{3}} U_{3} \\
\ddot{\psi}=J_{z_{2}} \dot{\varphi} \dot{\vartheta}+J_{z_{3}} U_{4}
\end{array}\right\}
$$

where $£_{x}=\sin \varphi \sin \psi+\sin \varphi \vartheta \cos \cos \psi, £_{y}=\cos \varphi \sin \vartheta \sin \psi-\sin \varphi \cos \psi$, $£_{z}=\cos \varphi \cos \vartheta, b_{m}=b / m, J_{x_{3}}=b l / I_{x}, J_{y_{3}}=b l / I_{y}, J_{z_{3}}=1 / I_{z}, J_{x_{2}}=\left(I_{y}-\right.$ $\left.I_{z}\right) / I_{x}, J_{x_{1}}=I_{R} / I_{x}, J_{y_{2}}=\left(I_{z}-I_{x}\right) / I_{y}, J_{y_{1}}=-I_{R} / I_{y}$ and $J_{z_{2}}=\left(I_{x}-I_{y}\right) / I_{z}$. The typical model parameters are the inertia constants $\left(I_{x}, I_{y}, I_{z}, I_{r}\right)$, position states $(x, y, z, \varphi, \vartheta, \psi)$, thrust coefficient $(b)$, arm's length $(l)$, quadcopter mass $(m)$ and drag factor $(d)$. The control inputs are $U_{1}=\sum_{i=1}^{4} \mho_{i}^{2}, U_{2}=$ $-\mho_{2}^{2}+\mho_{4}^{2}, U_{3}=\mho_{1}^{2}-\mho_{3}^{2}$, and $U_{4}=\sum_{i=1}^{4}(-1)^{i} \mho_{i}^{2}$. It is evident that control inputs $U_{2}, U_{3}$ and $U_{4}$ derive $\varphi, \vartheta$ and $\psi$ angles, respectively, whereas the control input $U_{1}$ controls three configuration variables $(x, y, z)$, which is not an easy task. Therefore, the following coordinates conversion will transform the original model to a control convenient regular form.

$$
v=x-\Gamma_{x}, \quad \varrho=y-\Gamma_{y}
$$

Note that $\Gamma_{x}=\left(£_{x} / £_{z}\right) z$ and $\Gamma_{y}=\left(£_{y} / £_{z}\right) z$ are the solutions of $d\left(\Gamma_{x}\right) / d z=$ $£_{x} / £_{z}$ and $d\left(\Gamma_{y}\right) / d z=£_{y} / £_{z}$.

The time differentiation of (54) can be evaluated in the following form.

$$
\begin{aligned}
& \dot{v}=\dot{x}-\frac{£_{x}}{£_{z}} \dot{z}, \quad \ddot{v}=\ddot{x}-\frac{£_{x}}{£_{z}} \ddot{z} \\
& \dot{\varrho}=\dot{y}-\frac{£_{y}}{£_{z}} \dot{z}, \quad \ddot{\varrho}=\ddot{y}-\frac{£_{y}}{£_{z}} \ddot{z}
\end{aligned}
$$

After invoking $\ddot{x}, \ddot{y}$ and $\ddot{z}$ in (55), the regular form of the quadcopter system (53) can be achieved as follows

$$
\left.\begin{array}{ll}
\dot{z}_{1}=z_{2}, & \dot{z}_{2}=-g+b_{m} £_{z} U_{1} \\
\dot{\psi}_{1}=\psi_{2}, & \dot{\psi}_{2}=J_{z_{2}} \vartheta_{2} \varphi_{2}+J_{z_{3}} U_{4} \\
\dot{\varrho}_{1}=\varrho_{2}, & \dot{\varrho}_{2}=£_{y} / £_{z}=g £_{\varrho} \\
\dot{\varphi}_{1}=\varphi_{2}, & \dot{\varphi}_{2}=J_{x_{2}} \psi_{2} \vartheta_{2}+J_{x_{1}} \bar{\mho} \vartheta_{2}+J_{x_{3}} U_{2} \\
\dot{v}_{1}=v_{2}, & \dot{v}_{2}=g £_{x} / £_{z}=g £_{v} \\
\dot{\vartheta}_{1}=\vartheta_{2}, & \dot{\vartheta}_{2}=J_{y_{2}} \psi_{2} \varphi_{2}+J_{y_{1}} \bar{\mho} \varphi_{2}+J_{y_{3}} U_{3}
\end{array}\right\}
$$


It is evidant that the system (56) have two fully-actuated subsystems $(z, \psi)$ and two under-actuared subsystems $(\varrho-\varphi, v-\vartheta)$. Since the internal dynamics $(x$ and $y)$ are interconnected with $\varphi$ and $\vartheta$-dynamics in practical scenario. Therefore, these dynamics are indirectly controlled by control inputs $U_{2}$ and $U_{3}$.

Now, the system 56 is ready to design any control system for its stability.

\subsubsection{Control design}

Now, the complete flight control of the quadcopter UAV (56) is developed in two-part: fully-actuated and under-actuated. However, for simplicity's purpose, the detail control design of $\psi$-dynamics (in case of the fully-actuated subsystem) and $(\varrho-\varphi)$ dynamics (in case of the under-actuated subsystem) is presented.

Now, to control the $\psi$-dynamics, the following mismatch is defined.

$$
\varepsilon_{\psi}=\psi_{1}-\psi_{d}
$$

The sliding mode can be enforced against the following fast fixed-time terminal attractor to get the main tracking objective.

$$
\Upsilon_{\psi}=\dot{\xi}_{\psi}+\alpha_{\psi}\left|\xi_{\psi}\right|^{\kappa_{\psi}} \operatorname{sign}\left(\xi_{\psi}\right)+\beta_{\psi} \chi_{\psi}\left(\xi_{\psi}\right)
$$

The reachability law can be defined as follows

$$
\dot{\Upsilon}_{\psi}=-\bar{\alpha}_{\psi}\left|\Upsilon_{\psi}\right|^{\bar{\kappa}_{\psi}} \operatorname{sign}\left(\Upsilon_{\psi}\right)-\bar{\beta}_{\psi}\left|\Upsilon_{\psi}\right|^{\bar{\gamma}_{\psi}} \operatorname{sign}\left(\Upsilon_{\psi}\right)
$$

In the light of (58) and (59), the control input $U_{4}$ can be developed as follows

$$
\begin{aligned}
U_{4} & =\frac{1}{J_{z_{3}}}\left(-J_{z_{2}} \vartheta_{2} \varphi_{2}-\bar{\alpha}_{\psi}\left|\Upsilon_{\psi}\right|^{\bar{\kappa}_{\psi}} \operatorname{sign}\left(\Upsilon_{\psi}\right)-\bar{\beta}_{\psi}\left|\Upsilon_{\psi}\right|^{\bar{\gamma}_{\psi}} \operatorname{sign}\left(\Upsilon_{\psi}\right)+\ddot{\psi}_{d}\right. \\
& \left.-\alpha_{\psi} \kappa_{\psi}\left|\xi_{\psi}\right|^{\kappa_{\psi}-1} \dot{\xi}_{\psi}-\beta_{\psi} \dot{\chi}_{\psi}\left(\xi_{\psi}\right)\right)
\end{aligned}
$$

Note that the control law (60) along sliding surface (58) will ensure the fast convergence of $\psi$-dynamics in fixed-time. Consequently, the mismatch $\xi_{\psi}$ will approach to zero in fixed-time [12].

Similarly, the proposed terminal sliding manifold is used to develop the following control law to control $z$-dynamics.

$$
\begin{aligned}
U_{1} & =\frac{1}{b_{m} \cos \vartheta_{1} \cos \varphi_{1}}\left(g-\bar{\alpha}_{z}\left|\Upsilon_{z}\right|^{\bar{\kappa}_{z}} \operatorname{sign}\left(\Upsilon_{z}\right)-\bar{\beta}_{z}\left|\Upsilon_{z}\right|^{\bar{\gamma}_{z}} \operatorname{sign}\left(\Upsilon_{z}\right)\right. \\
& \left.+\ddot{z}_{d}-\alpha_{z} \kappa_{z}\left|\xi_{z}\right|^{\kappa_{z}-1} \dot{\xi}_{z}-\beta_{z} \dot{\chi}_{z}\left(\xi_{z}\right)\right)
\end{aligned}
$$

Thus, the control design for the fixed-time stabilization of fully-actuated dynamics is completed. Now, before proceeding to controller design for the underactuated subsystem, the following remark is presented. 
Remark 6 Here, the main objective of the control design is to track $v(t)$ on the desired trajectory $v_{d}(t)$ in fixed-time while steering $\vartheta$ at the origin. To meet this objective, the terminal attractor can be established in following form.

$$
\$_{v}=\dot{\xi}_{v}+\alpha_{1}\left|\xi_{v}\right|^{\kappa_{1}} \operatorname{sign}\left(\xi_{v}\right)+\beta_{1} \chi_{1}\left(\xi_{v}\right)
$$

along with

$$
g £_{v}-\ddot{v}_{d}=-\$_{v}
$$

and

$$
\Upsilon_{1}=g £_{v}-\ddot{v}_{d}+\$_{v}
$$

Since, $\dot{\vartheta}$ does not appeared in the first derivative of (64), therefore, the system relative degree can be increased by introducing the following hierarchical manifold.

$$
\Upsilon_{2}=\dot{\Upsilon}_{1}+\alpha_{2}\left|\Upsilon_{1}\right|^{\kappa_{2}} \operatorname{sign}\left(\Upsilon_{1}\right)+\beta_{2} \chi_{2}\left(\Upsilon_{1}\right)
$$

According to remark 5, the reachability law is presented as follows

$$
\dot{\Upsilon}_{2}=-\bar{\alpha}_{2}\left|\Upsilon_{2}\right|^{\bar{\kappa}_{2}} \operatorname{sign}\left(\Upsilon_{2}\right)-\bar{\beta}_{2}\left|\Upsilon_{2}\right|^{\bar{\gamma}_{2}} \operatorname{sign}\left(\Upsilon_{2}\right)
$$

where $\bar{\kappa}_{1 v}$ and $\bar{\kappa}_{2 v}$ are positive design constants. Following the theorem 1 , the following control input can ensure the sliding mode establishment against the proposed fixed-time terminal attractor (65).

$$
\begin{aligned}
& U_{3}=\frac{1}{J_{y_{3}}}\left(\frac { - 1 } { g \frac { \partial £ _ { v } } { \partial \vartheta } } \left(g\left(\frac{\partial £_{v}}{\partial \varphi} \ddot{\varphi}+\frac{\partial £_{v}}{\partial \psi} \ddot{\psi}+\frac{d}{d t}\left(\frac{\partial £_{v}}{\partial \vartheta}\right) \dot{\vartheta}+\frac{d}{d t}\left(\frac{\partial £_{v}}{\partial \varphi}\right) \dot{\varphi}+\frac{d}{d t}\left(\frac{\partial £_{v}}{\partial \psi}\right) \dot{\psi}\right)\right.\right. \\
& -\dddot{v}_{d}+\ddot{\$}_{v}+\alpha_{2}\left(\kappa_{2}\right)\left|\Upsilon_{1}\right|^{\kappa_{2}-1} \dot{\Upsilon}_{1}+\beta_{2} \dot{\chi}_{2}\left(\Upsilon_{1}\right)+\bar{\alpha}_{2}\left|\Upsilon_{2}\right|^{\bar{\kappa}_{2}} \operatorname{sign}\left(\Upsilon_{2}\right) \\
& \left.\left.+\bar{\beta}_{2}\left|\Upsilon_{2}\right|^{\bar{\gamma}_{2}} \operatorname{sign}\left(\Upsilon_{2}\right)\right)-\left(J_{y_{2}} \psi_{2} \varphi_{2}+J_{y_{1}} \bar{\mho} \varphi_{2}\right)\right)
\end{aligned}
$$

When the sliding mode is established, then $\Upsilon_{2} \rightarrow 0$ leads eventually to assure the tracking error $\xi_{v}$ enforcement to zero in fixed-time.

In similar fashion, the following control scheme can be designed to control the other under-actuated subsystem (of $y$ and $\varphi$ dynamics).

$$
\begin{aligned}
& U_{2}=\frac{1}{J_{x_{3}}}\left(\frac { - 1 } { g \frac { \partial £ _ { \varrho } } { \partial \varphi } } \left(g\left(\frac{\partial £_{\varrho}}{\partial \vartheta} \ddot{\vartheta}+\frac{\partial £_{\varrho}}{\partial \psi} \ddot{\psi}+\frac{d}{d t}\left(\frac{\partial £_{\varrho}}{\partial \vartheta}\right) \dot{\vartheta}+\frac{d}{d t}\left(\frac{\partial £_{\varrho}}{\partial \varphi}\right) \dot{\varphi}+\frac{d}{d t}\left(\frac{\partial £_{\varrho}}{\partial \psi}\right) \dot{\psi}\right)\right.\right. \\
& -\dddot{\varrho}_{d}+\ddot{\$}_{y}+\alpha_{2}\left(\kappa_{2}\right)\left|\Upsilon_{1}\right|^{\kappa_{2}-1} \dot{\Upsilon}_{1}+\beta_{2} \dot{\chi}_{2}\left(\Upsilon_{1}\right)+\bar{\alpha}_{2}\left|\Upsilon_{2}\right|^{\bar{\kappa}_{2}} \operatorname{sign}\left(\Upsilon_{2}\right) \\
& \left.\left.+\bar{\beta}_{2}\left|\Upsilon_{2}\right|^{\bar{\gamma}_{2}} \operatorname{sign}\left(\Upsilon_{2}\right)\right)-\left(J_{x_{2}} \psi_{2} \vartheta_{2}+J_{x_{1}} \bar{\mho} \vartheta_{2}\right)\right)
\end{aligned}
$$

Remark 7 It is worthy of mentioning that the approximation of nonlinear drift terms is accomplished via feed-forward neural networks. In simulation, the words $£_{\varrho}$ and $£_{v}$ should be replaced by respective estimates $\hat{£}_{\varrho}$ and $\hat{£}_{v}$.

Thus, the discussion on the control of under-actuated subsystems is finished. 


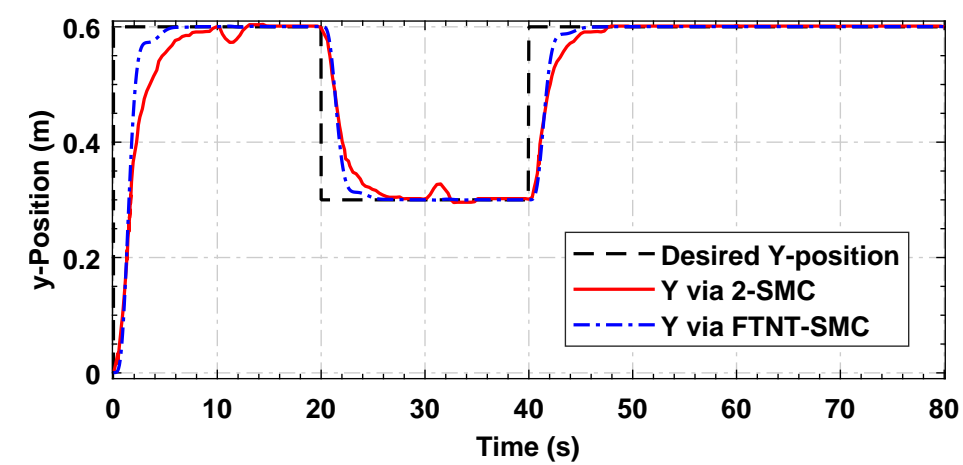

Fig. 6 The tracking performance of x-subsystem via the proposed control law in comparison with standard literature [31]

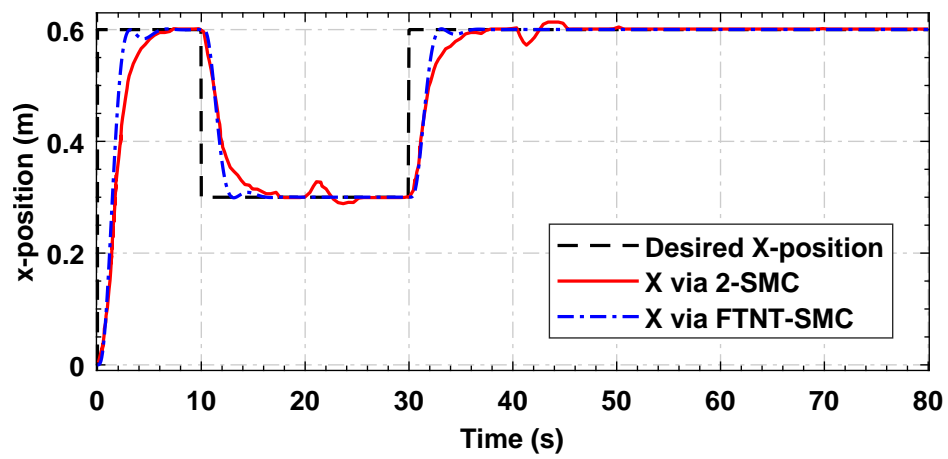

Fig. 7 The tracking performance of y-subsystem via the proposed control law in comparison with standard literature [31]

\subsubsection{Result Discussion}

Numerical simulations in the Matlab/ Simulink environment are used to validate the efficacy and supremacy of the proposed control algorithm in terms of robustness against unknown disturbances $\Delta=K_{\Delta} \sin (2 \pi t)$ and trajectory tracking in high precision. In addition, the RBF-based neural network is used to estimate the nonlinear drift functions of the under-actuated subsystem. The developed control algorithm simulations are compared to those of a standard fractional-order SMC scheme (designed in [31]). The model parameters for the quadcopter that have been used in simulation are as follows: the thrust coefficient is $b=3.13 \times 10^{-5} N . s^{2}$, the arm length $l=0.23 \mathrm{~m}$, the quadcopter's mass is $m=0.650 \mathrm{Kg}$, the inertia coefficients are $J_{x}=J_{y}=J_{z}=7.5 \times 10^{-3}$ $\mathrm{kg} . \mathrm{m} 2$, the drag coefficients are $d=7.5 \times 10^{-7} N . m . s^{2}$, and the rotor inertia coefficient is $J_{r}$. For simulation tests, $\left[\begin{array}{lll}0 & 0 & 0\end{array}\right] \mathrm{m}$ and $\left[\begin{array}{lll}0 & 0 & 0\end{array}\right] \mathrm{rad}$ are the initial values of translational positions and angular orientations. Meanwhile, the trial and error method is used for the selection of controller gains. 


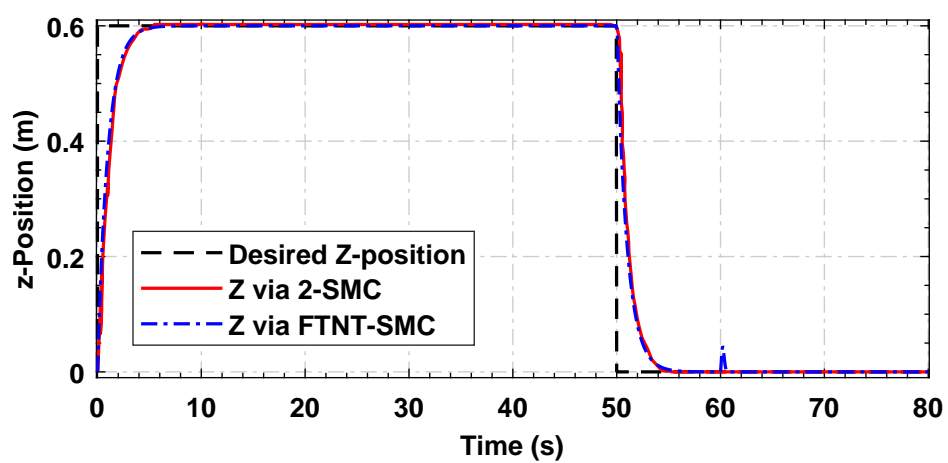

Fig. 8 The tracking performance of z-subsystem via the proposed control law in comparison with standard literature [31]

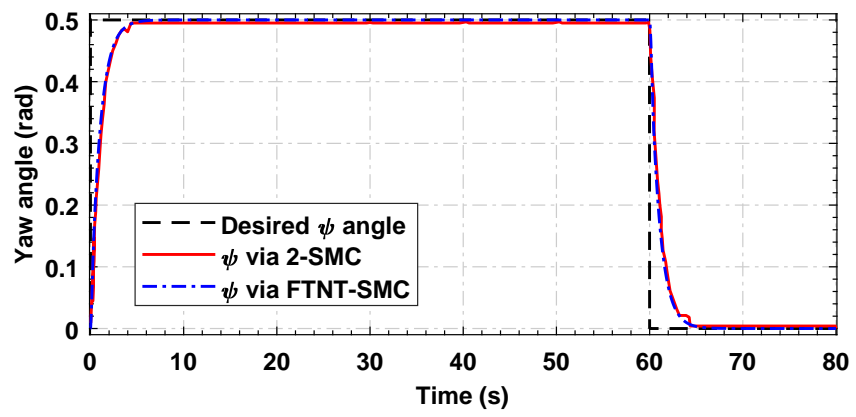

Fig. 9 Roll angle trajectory performance of the proposed control law in comparison with standard literature [31]

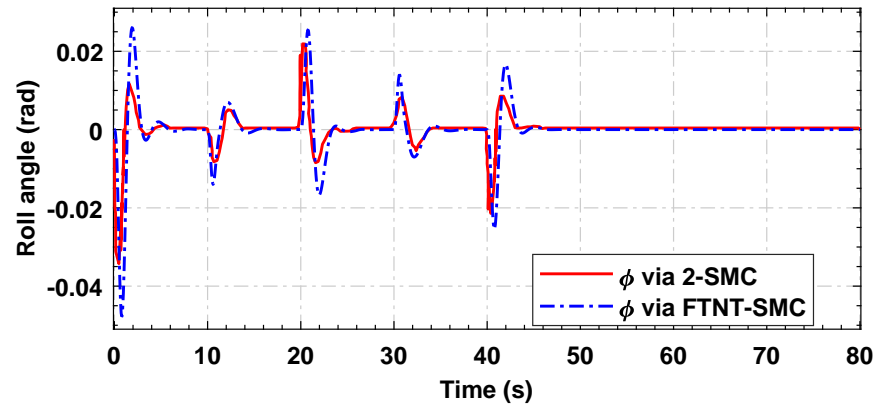

Fig. 10 Pitch angle trajectory performance of the proposed control law in comparison with standard literature [31]

Figure 6-8 shows the tracking performance of the quadcopter system under the newly configured control algorithm. The proposed controller enforces the tracking error to zero with a slightly faster-tracking speed than [31]. Meanwhile, figure 9-11 depicts the stabilization of roll, pitch, and yaw angles at origin, which further assure that the configured flight controller produces a significantly faster converging response with less overshoot than its counter- 


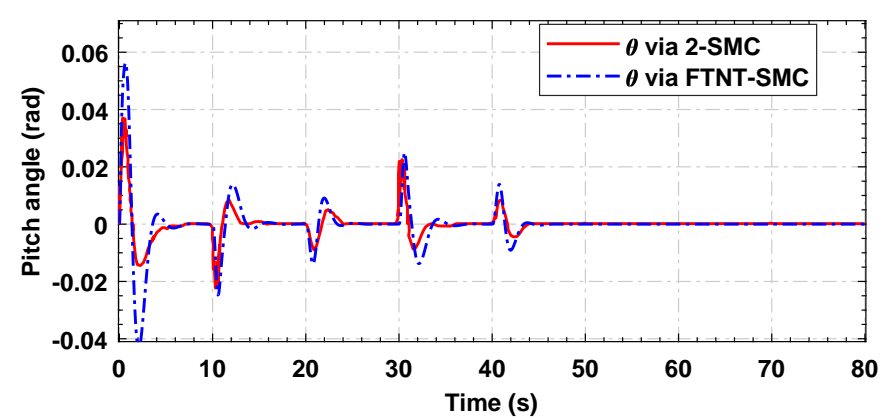

Fig. 11 Yaw angle trajectory performance of the proposed control law in comparison with standard literature [31]

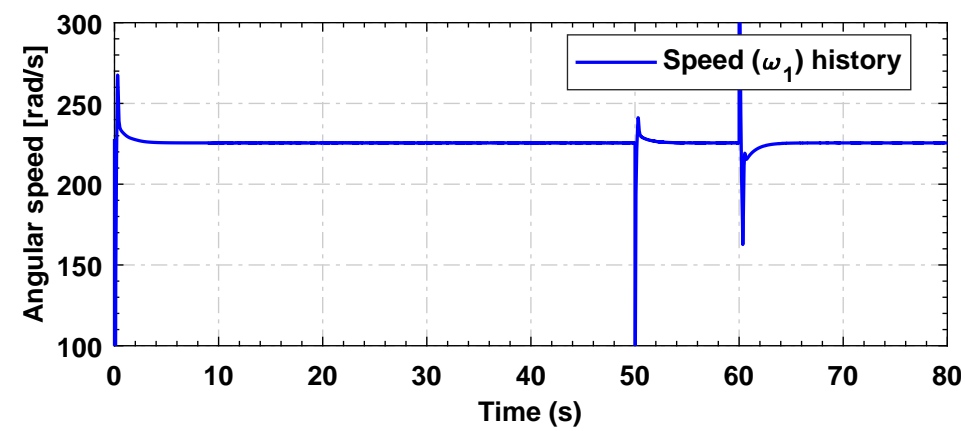

Fig. 12 The time history of control input $\omega_{1}$ produced by the proposed control law

part presented in [31]. In addition, using the designed control method eliminates the problem of high-frequency chattering in conventional sliding modes. In addition, the time histories of the applied control inputs $\omega_{1}, \omega_{2}, \omega_{3}$ and $\omega_{4}$ under the proposed control protocol are depicted in figures 13, from which it is clear that the control inputs are substantially smooth and behave in a very feasible manner. Furthermore, the configured control scheme with applied control inputs suppressed unwanted external uncertainties, resulting in the output tracking error being reduced to zero in a fixed period of time. The overall theoretical and numerical study of both cart-pendulum and quadcopter UAV concludes that the newly proposed control method is one of the best candidates for controlling the considered class (which can be transformed in its equivalent regular form).

\section{Conclusion}

A non-singular fast terminal sliding mode-based neuro-adaptive control synthesis is developed for a class of UNS in this research work. Some non-singular coordinates are presented to transform the generalized mathematical model of the considered class to a control convenient regular form to pursue the design. The drift function estimations via RBF neural network are introduced 


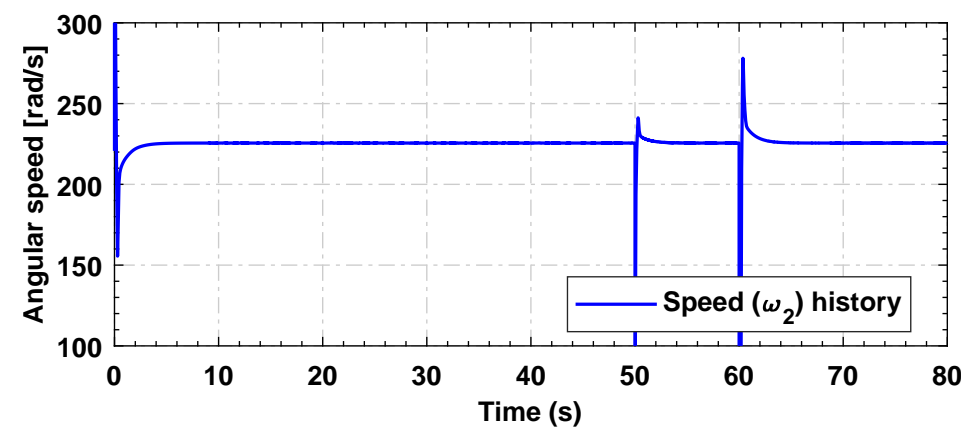

Fig. 13 The time history of control input $\omega_{2}$ produced by the proposed control law

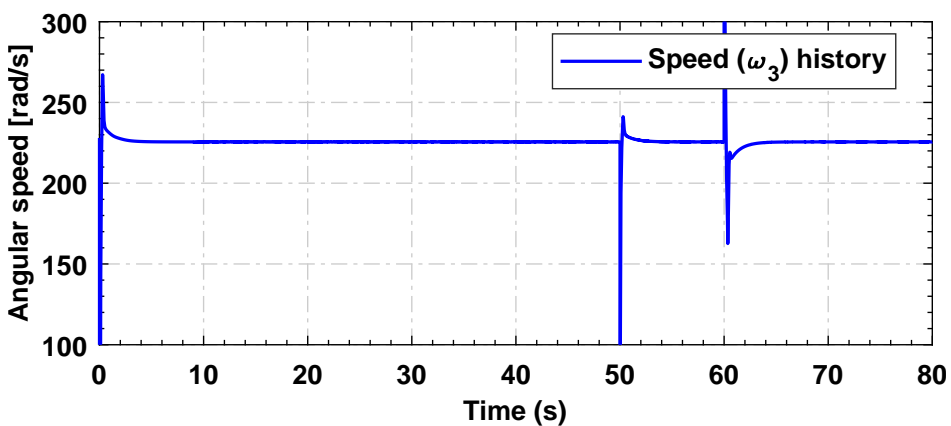

Fig. 14 The time history of control input $\omega_{3}$ produced by the proposed control law

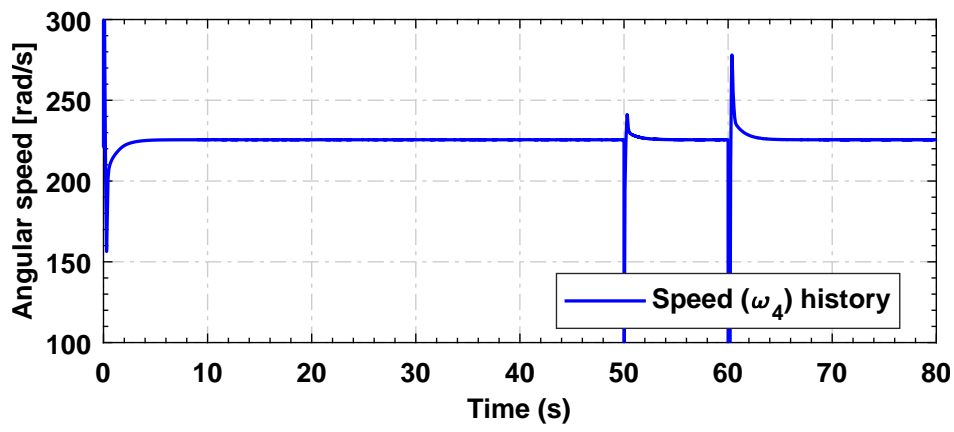

Fig. 15 The time history of control input $\omega_{4}$ produced by the proposed control law

to improve the newly configured control system's robustness. A non-singular fast TSMC synthesis is devised for the formulated problem, which is free from the singularity problem and has minimum settling time without knowing the initial conditions. The fixed-time stability of both system states and sliding manifolds is ensured with the help of Lyapunov stability theory. The simulation results of quadcopter and cart-pendulum systems under the proposed control algorithm are compared with their respective standard literature [31] 
and [21] to comprehensively demonstrate the feasibility and benefits of the proposed synthesis over the existing standard literature.

\section{Conflict of interest}

The authors declare that they have no conflict of interest.

\section{References}

1. Almutairi, N.B., Zribi, M.: On the sliding mode control of a ball on a beam system. Nonlinear dynamics 59(1-2), 221 (2010)

2. Bartolini, G., Ferrara, A., Usai, E.: Chattering avoidance by second-order sliding mode control. IEEE Transactions on automatic control 43(2), 241-246 (1998)

3. Corradini, M.L., Cristofaro, A.: Nonsingular terminal sliding-mode control of nonlinear planar systems with global fixed-time stability guarantees. Automatica 95, 561-565 (2018)

4. ud Din, S., ur Rehman, F., Khan, Q.: Smooth super-twisting sliding mode control for the class of underactuated systems. PloS one 13(10), e0203667 (2018)

5. Din, S.U., Khan, Q., Rehman, F., Akmeliawanti, R.: A comparative experimental study of robust sliding mode control strategies for underactuated systems. IEEE Access 5, 10068-10080 (2017). DOI 10.1109/ACCESS.2017.2712261

6. Fantoni, I., Lozano, R.: Non-linear control for underactuated mechanical systems. Springer Science \& Business Media (2002)

7. Fessi, R., Bouallègue, S., Haggège, J., Vaidyanathan, S.: Terminal sliding mode controller design for a quadrotor unmanned aerial vehicle. In: Applications of Sliding Mode Control in Science and Engineering, pp. 81-98. Springer (2017)

8. Ghommam, J., Mnif, F., Benali, A., Derbel, N.: Asymptotic backstepping stabilization of an underactuated surface vessel. IEEE Transactions on Control Systems Technology 14(6), 1150-1157 (2006)

9. Gruszka, A., Malisoff, M., Mazenc, F.: On tracking for the pvtol model with bounded feedbacks. In: American Control Conference (ACC), 2011, pp. 1428-1433. IEEE (2011)

10. Huang, X., Yan, Y.: Saturated backstepping control of underactuated spacecraft hovering for formation flights. IEEE Transactions on Aerospace and Electronic Systems 53(4), 1988-2000 (2017)

11. Huang, Y., Jia, Y.: Fixed-time consensus tracking control for second-order multi-agent systems with bounded input uncertainties via nfftsm. IET Control Theory \& Applications 11(16), 2900-2909 (2017)

12. Khan, Q., Akmeliawati, R., Bhatti, A.I., Ashraf, M.: Robust stabilization of underactuated nonlinear systems: A fast terminal sliding mode approach. ISA transactions 66 , 241-248 (2017)

13. Lee, S.G., et al.: Sliding mode controls of double-pendulum crane systems. Journal of Mechanical Science and Technology 27(6), 1863-1873 (2013)

14. Levant, A.: Quasi-continuous high-order sliding-mode controllers. In: Decision and Control, 2003. Proceedings. 42nd IEEE Conference on, vol. 5, pp. 4605-4610. IEEE (2003)

15. Li, B., Qin, K., Xiao, B., Yang, Y.: Finite-time extended state observer based fault tolerant output feedback control for attitude stabilization. ISA transactions 91, 11-20 (2019)

16. Li, T., Zhao, H.: Global finite-time adaptive control for uncalibrated robot manipulator based on visual servoing. ISA transactions 68, 402-411 (2017)

17. Mofid, O., Mobayen, S.: Adaptive sliding mode control for finite-time stability of quadrotor uavs with parametric uncertainties. ISA transactions 72, 1-14 (2018)

18. Ni, J., Liu, L., Liu, C., Hu, X., Li, S.: Fast fixed-time nonsingular terminal sliding mode control and its application to chaos suppression in power system. IEEE Transactions on Circuits and Systems II: Express Briefs 64(2), 151-155 (2016) 
19. Pan, B., Fareed, U., Qing, W., Tian, S.: A novel fractional order pid navigation guidance law by finite time stability approach. ISA transactions 94, 80-92 (2019)

20. Polyakov, A.: Nonlinear feedback design for fixed-time stabilization of linear control systems. IEEE Transactions on Automatic Control 57(8), 2106-2110 (2011)

21. Riachy, S., Orlov, Y., Floquet, T., Santiesteban, R., Richard, J.P.: Second-order sliding mode control of underactuated mechanical systems i: Local stabilization with application to an inverted pendulum. International Journal of Robust and Nonlinear Control: IFAC-Affiliated Journal 18(4-5), 529-543 (2008)

22. Soysal, B.: Real-time control of an automated guided vehicle using a continuous mode of sliding mode control. Turkish Journal of Electrical Engineering \& Computer Sciences 22(5), 1298-1306 (2014)

23. Talebi, H.A., Khorasani, K., Tafazoli, S.: A recurrent neural-network-based sensor and actuator fault detection and isolation for nonlinear systems with application to the satellite's attitude control subsystem. IEEE Transactions on Neural Networks 20(1), 45-60 (2008)

24. Tian, B., Lu, H., Zuo, Z., Wang, H.: Fixed-time stabilization of high-order integrator systems with mismatched disturbances. Nonlinear Dynamics 94(4), 2889-2899 (2018)

25. Tian, Y., Cai, Y., Deng, Y.: A fast nonsingular terminal sliding mode control method for nonlinear systems with fixed-time stability guarantees. IEEE Access 8, 60444-60454 (2020)

26. Ullah, S., Khan, Q., Mehmood, A., Bhatti, A.I.: Robust backstepping sliding mode control design for a class of underactuated electro-mechanical nonlinear systems. J. Electr. Eng. Technol. 15, 1821-1828 (2020). DOI https://doi.org/10.1007/s42835-02000436-3

27. Utkin, V., Guldner, J., Shi, J.: Sliding mode control in electro-mechanical systems. CRC press (2009)

28. Zhang, M., Tarn, T.J.: Hybrid control of the pendubot. IEEE/ASME transactions on mechatronics 7(1), 79-86 (2002)

29. Zhang, Y., Tang, S., Guo, J.: Adaptive terminal angle constraint interception against maneuvering targets with fast fixed-time convergence. International Journal of Robust and Nonlinear Control 28(8), 2996-3014 (2018)

30. Zhao, D., Zhu, Q., Dubbeldam, J.: Terminal sliding mode control for continuous stirred tank reactor. Chemical engineering research and design 94, 266-274 (2015)

31. Zheng, E.H., Xiong, J.J., Luo, J.L.: Second order sliding mode control for a quadrotor uav. ISA transactions 53(4), 1350-1356 (2014)

32. Zou, Y.: Nonlinear robust adaptive hierarchical sliding mode control approach for quadrotors. International Journal of Robust and Nonlinear Control 27(6), 925-941 (2017)

33. Zuo, Z.: Nonsingular fixed-time consensus tracking for second-order multi-agent networks. Automatica 54, 305-309 (2015) 
Figures

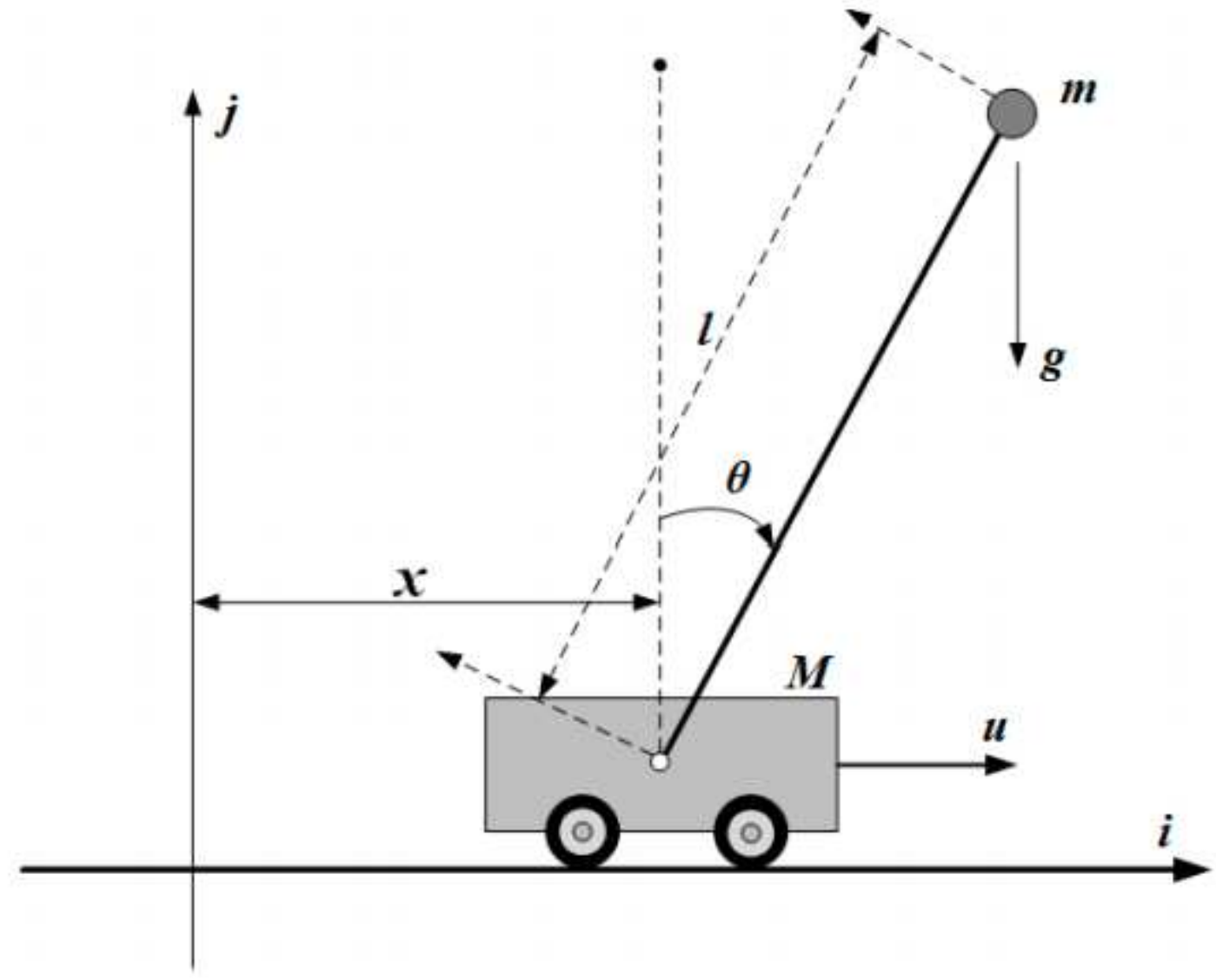

Figure 1

Cart-pendulum system

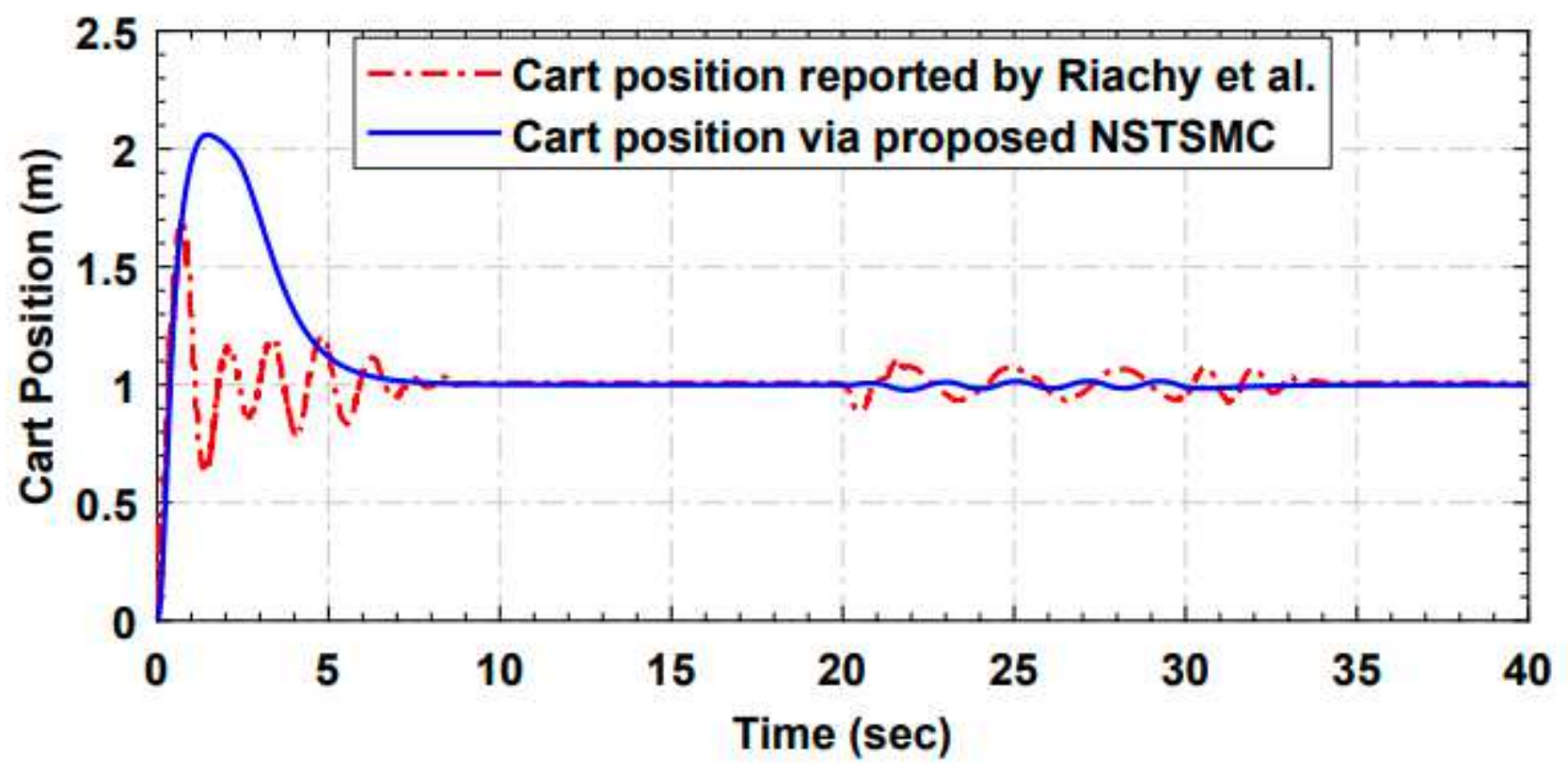

Figure 2 
The cart position tracking performance via the proposed control algorithm in comparison with standard literature [21]

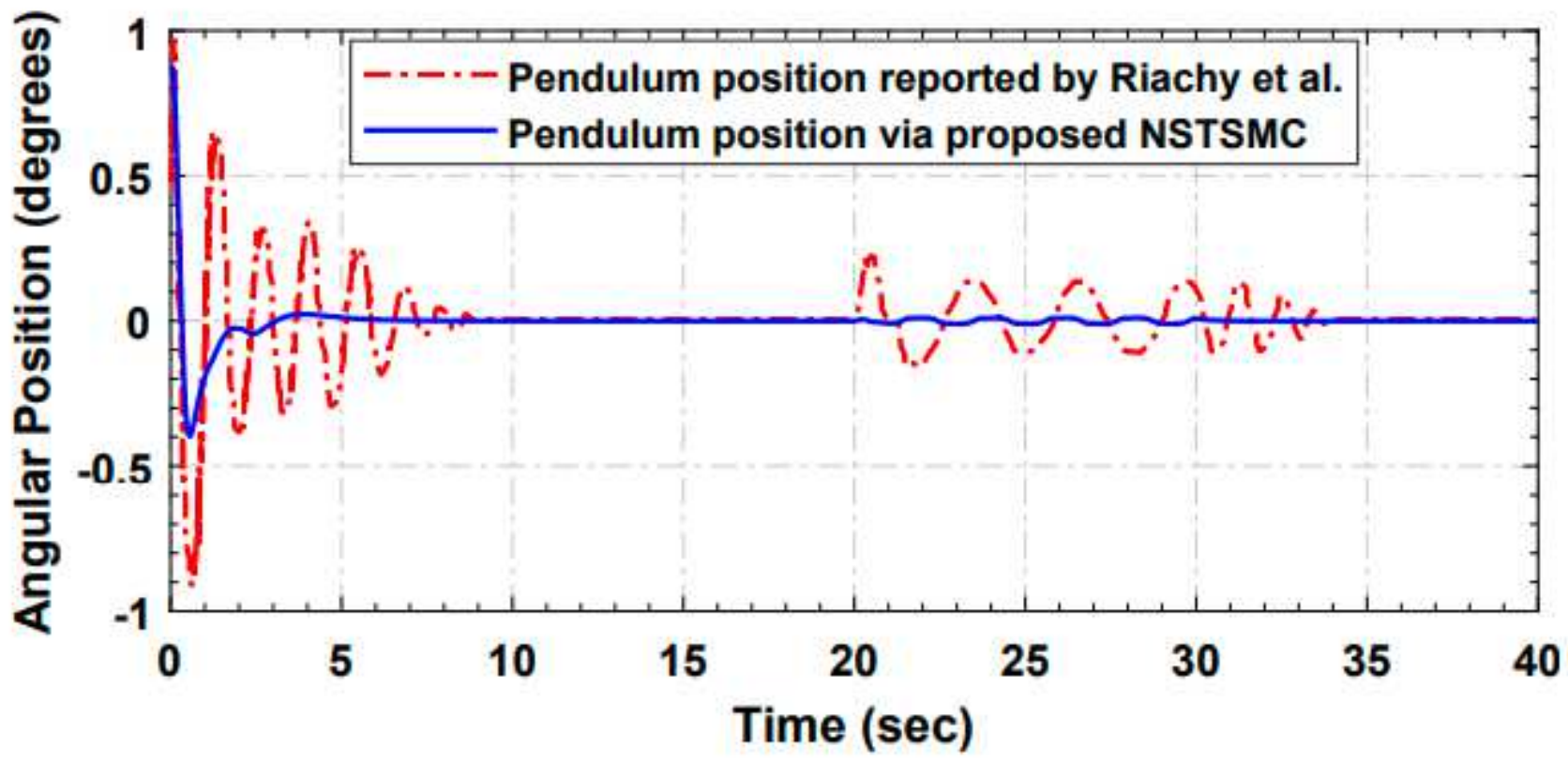

Figure 3

The rod angle stabilization performance via the proposed control algorithm in comparison with the standard literature [21]

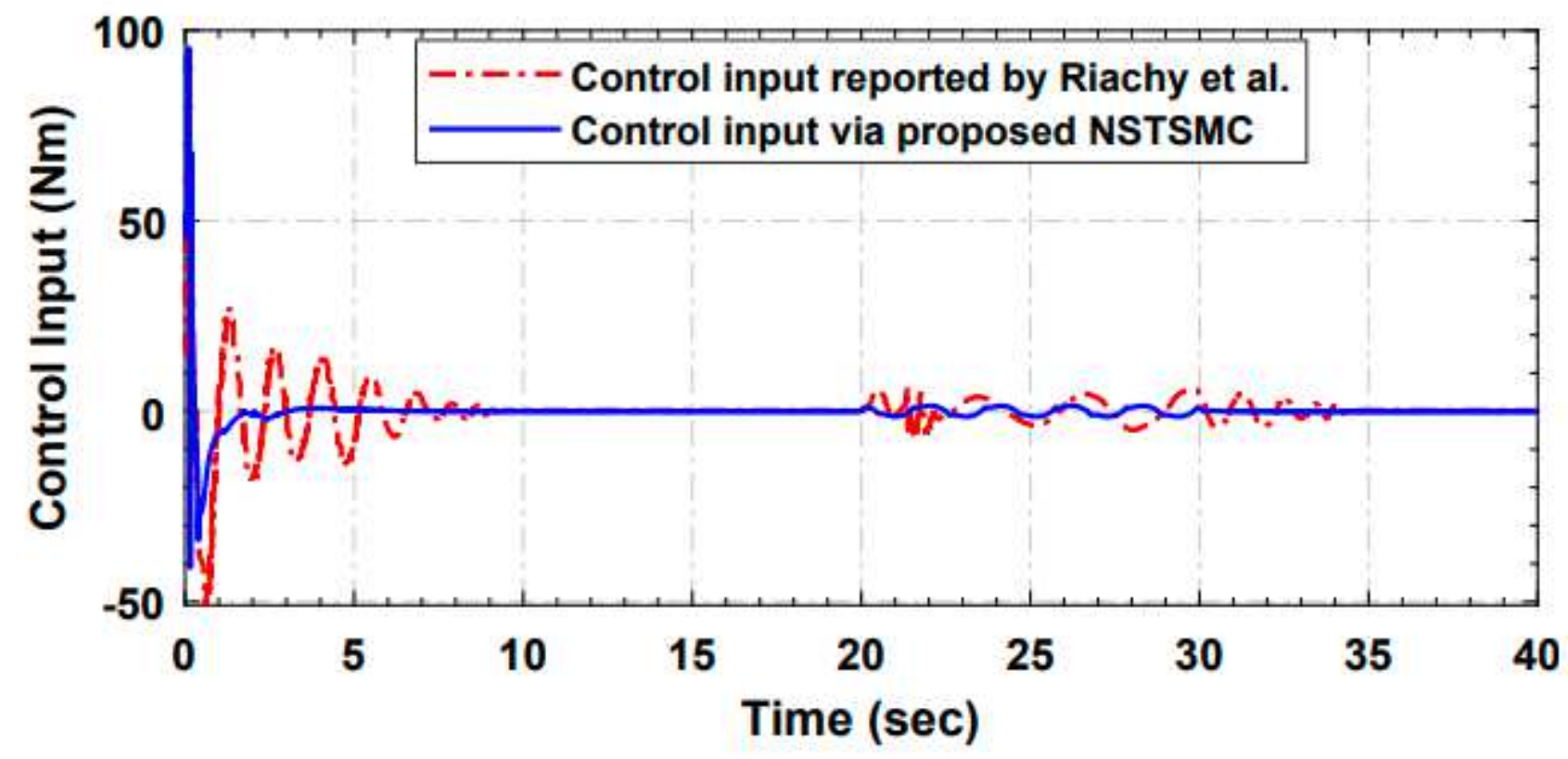

Figure 4

The control input history of the proposed control algorithm in comparison with the standard literature [21] 


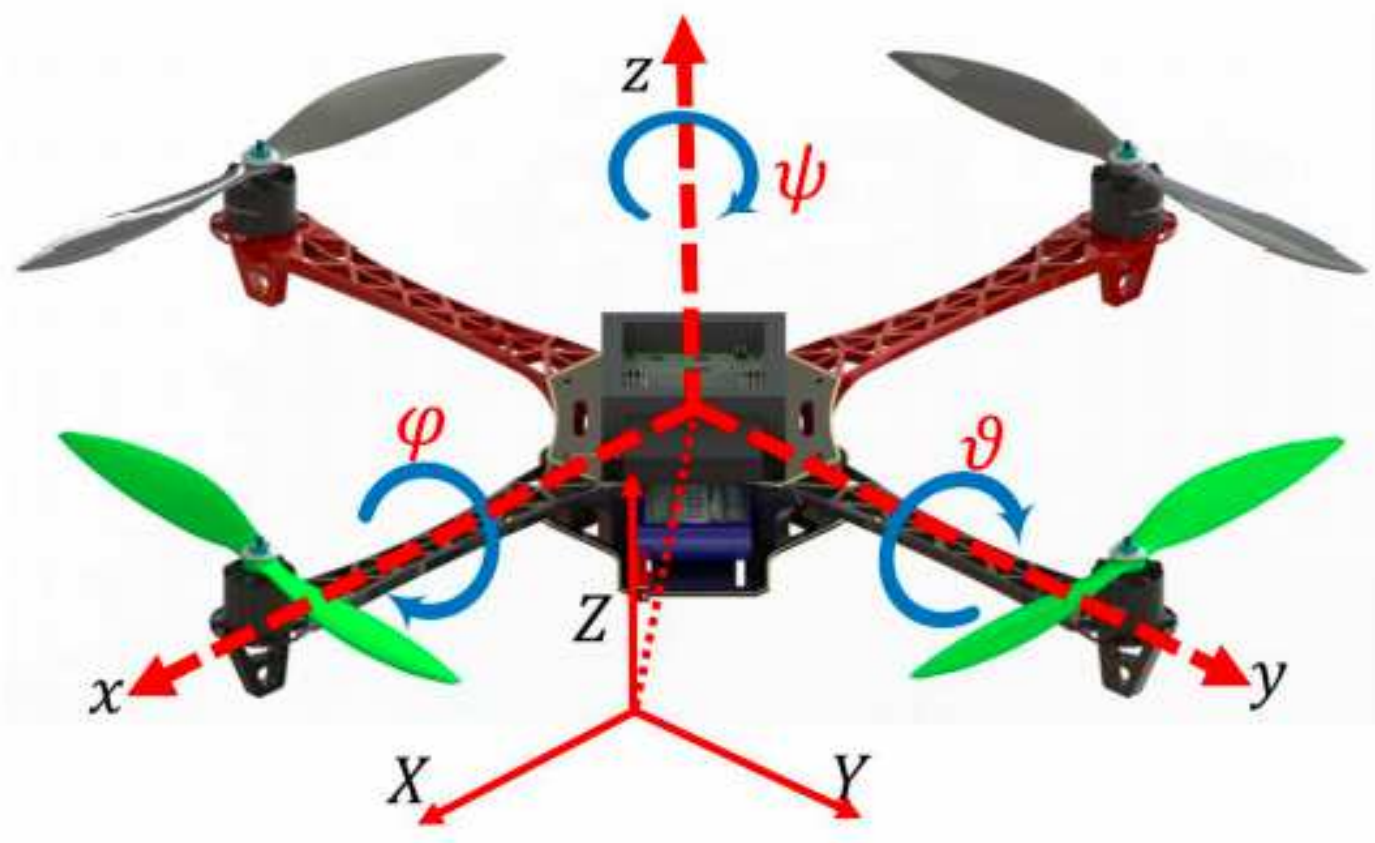

Figure 5

Quadcopter UAV

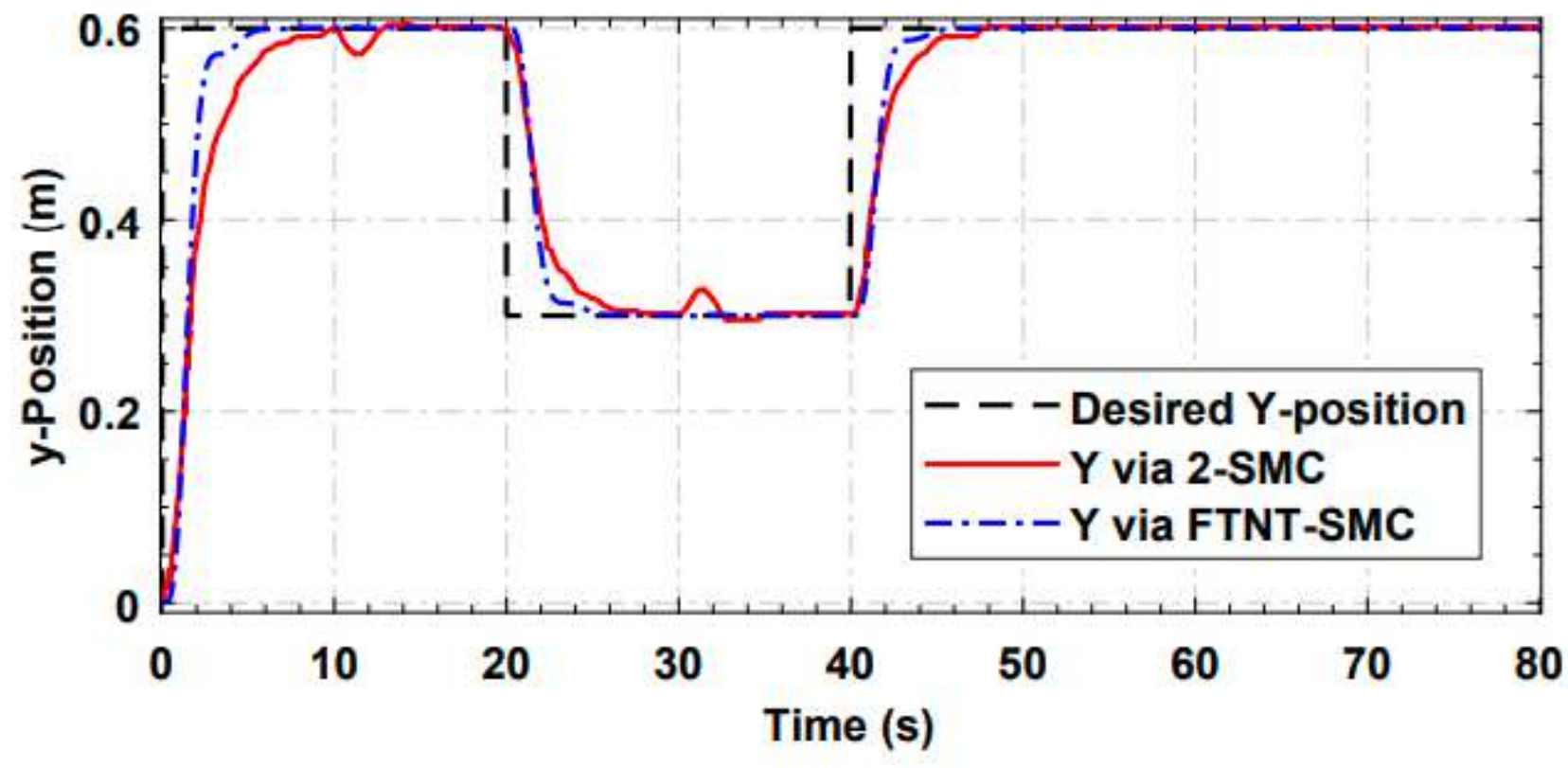

Figure 6

The tracking performance of $\mathrm{x}$-subsystem via the proposed control law in comparison with standard literature [31] 


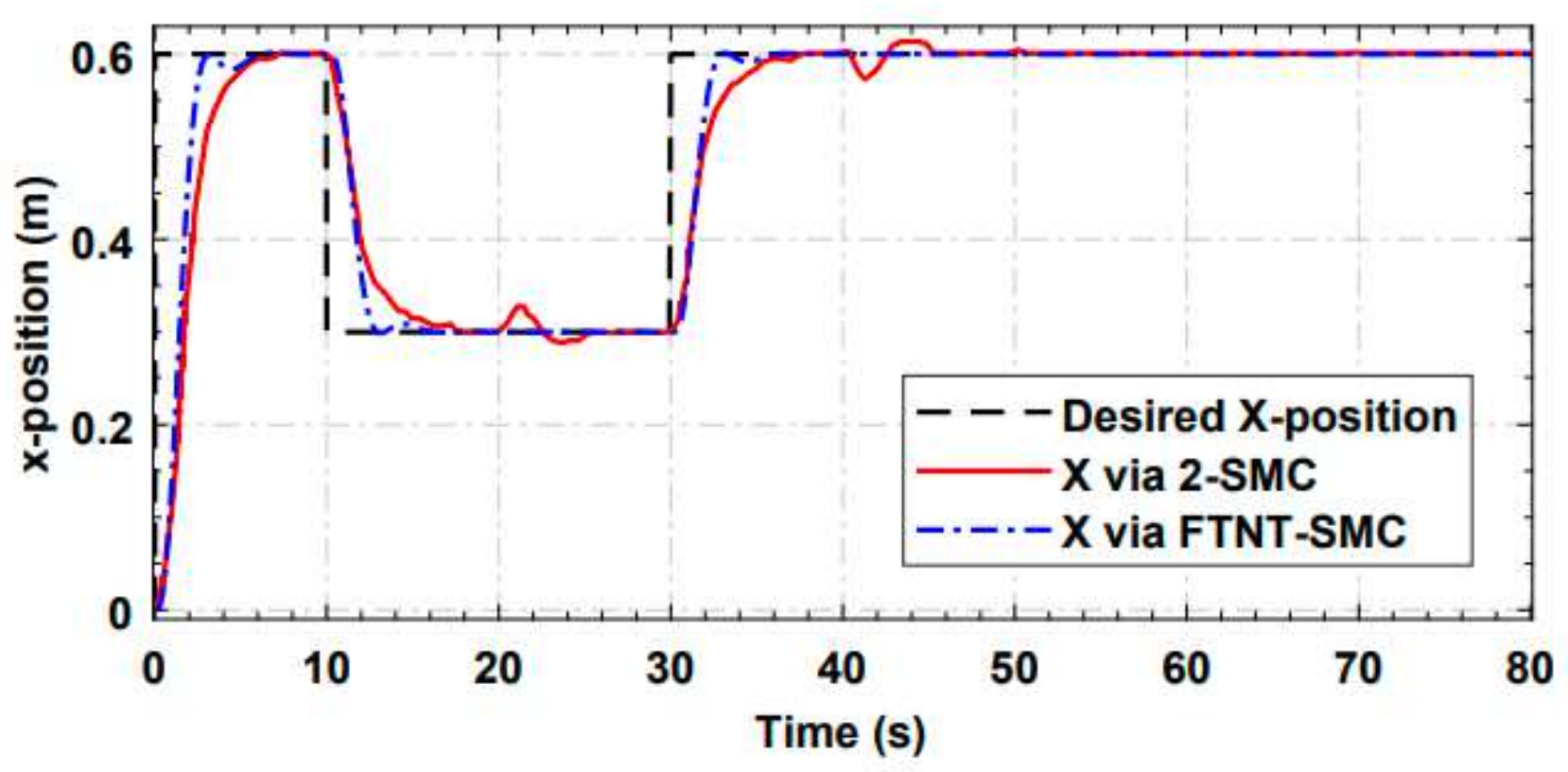

Figure 7

The tracking performance of y-subsystem via the proposed control law in comparison with standard literature [31]

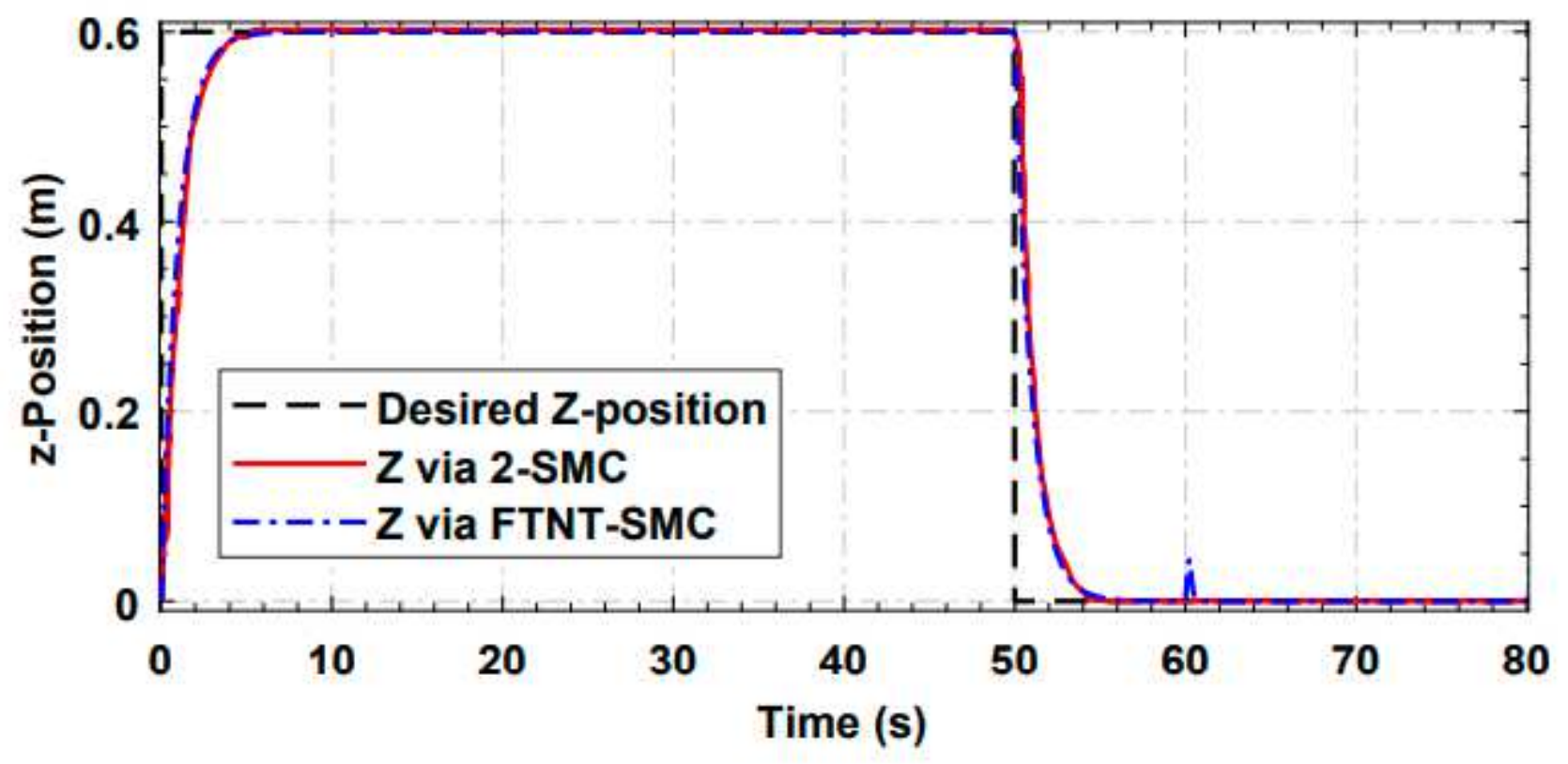

Figure 8

The tracking performance of z-subsystem via the proposed control law in comparison with standard literature [31] 


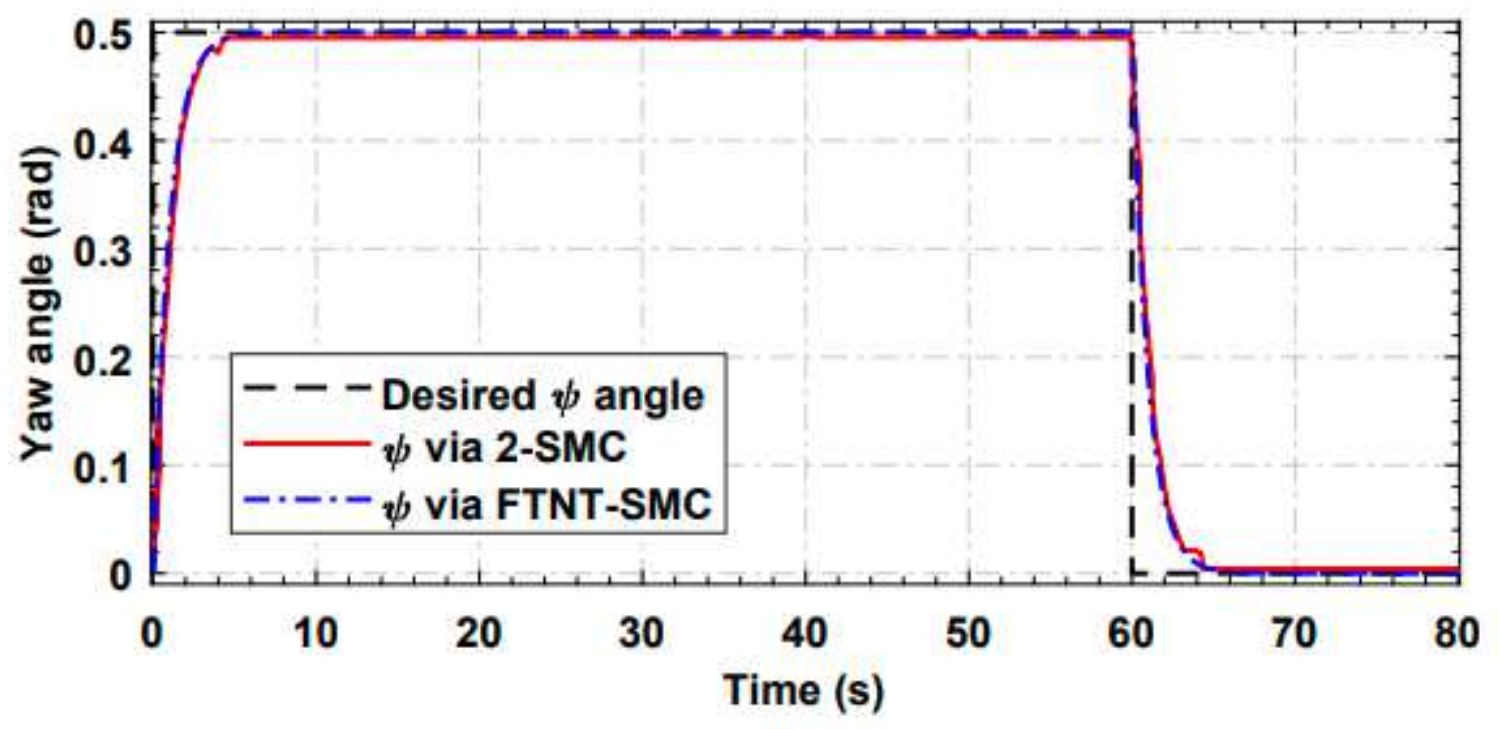

Figure 9

Roll angle trajectory performance of the proposed control law in comparison with standard literature [31]

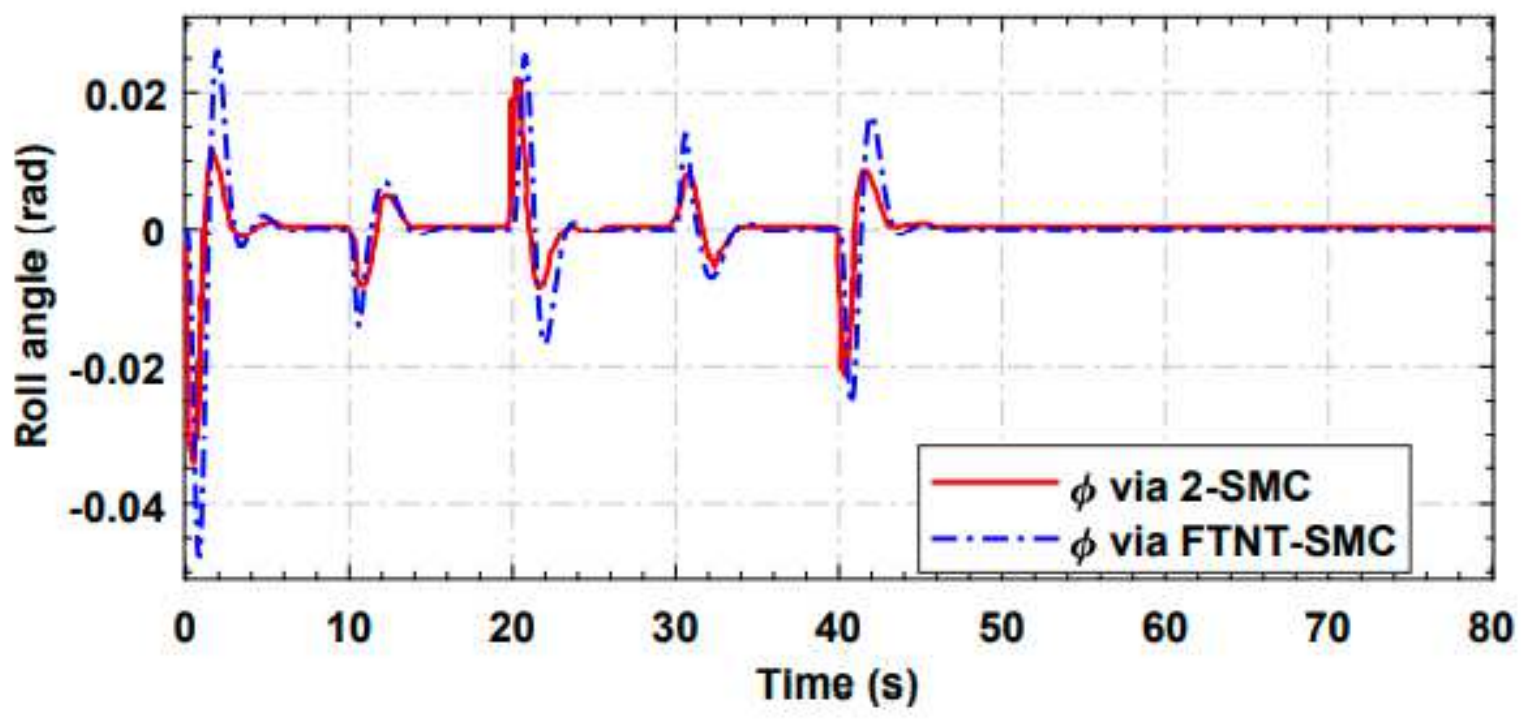

Figure 10

Pitch angle trajectory performance of the proposed control law in comparison with standard literature [31] 


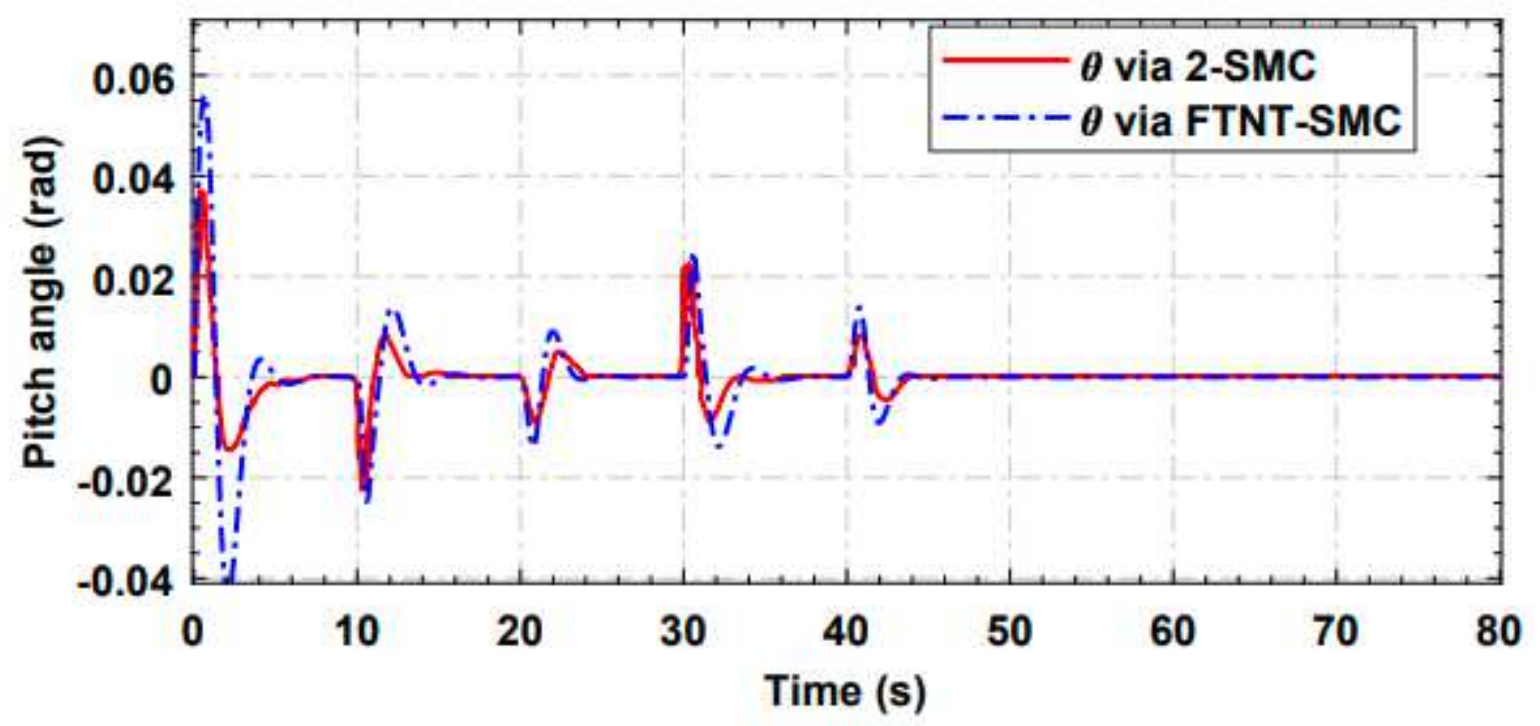

Figure 11

Yaw angle trajectory performance of the proposed control law in comparison with standard literature [31]

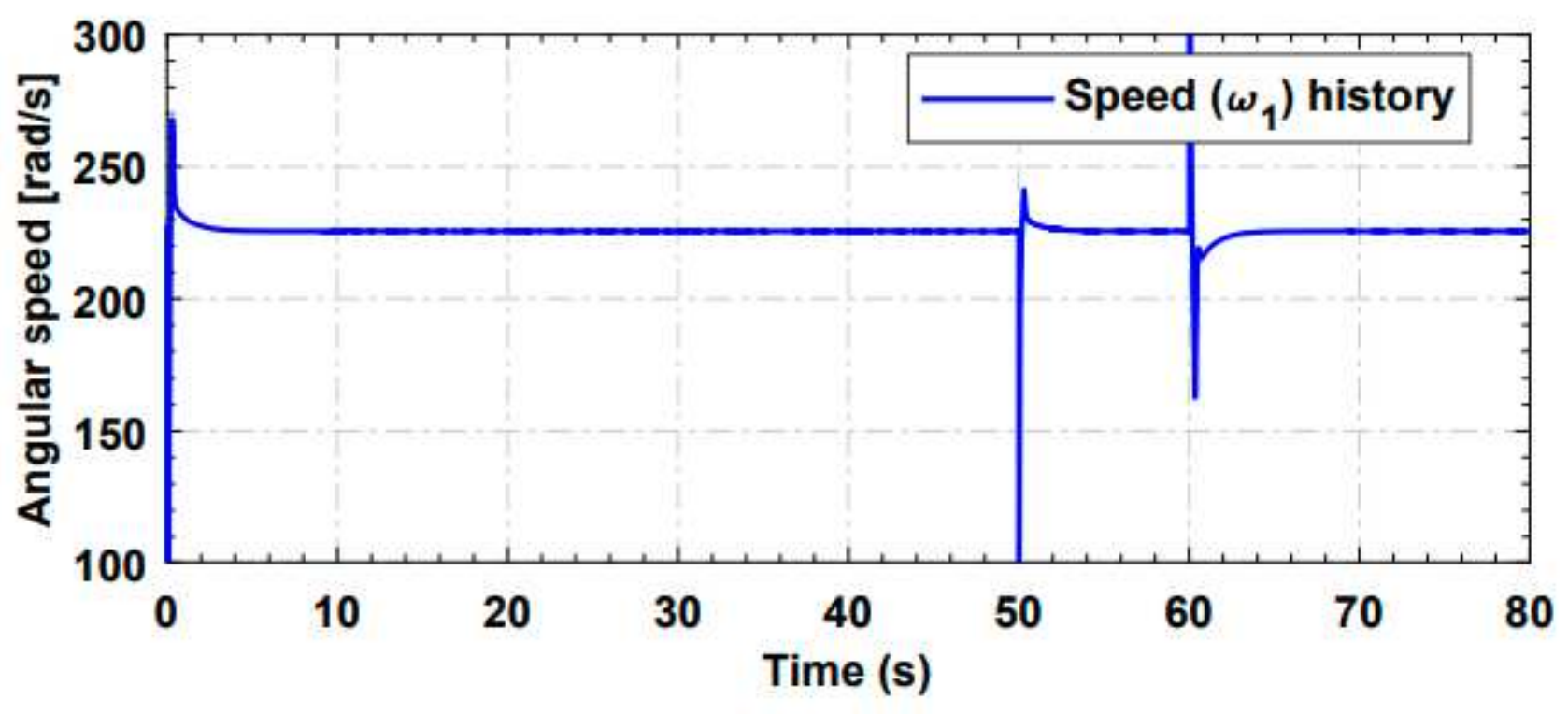

Figure 12

The time history of control input $\omega 1$ produced by the proposed control law 


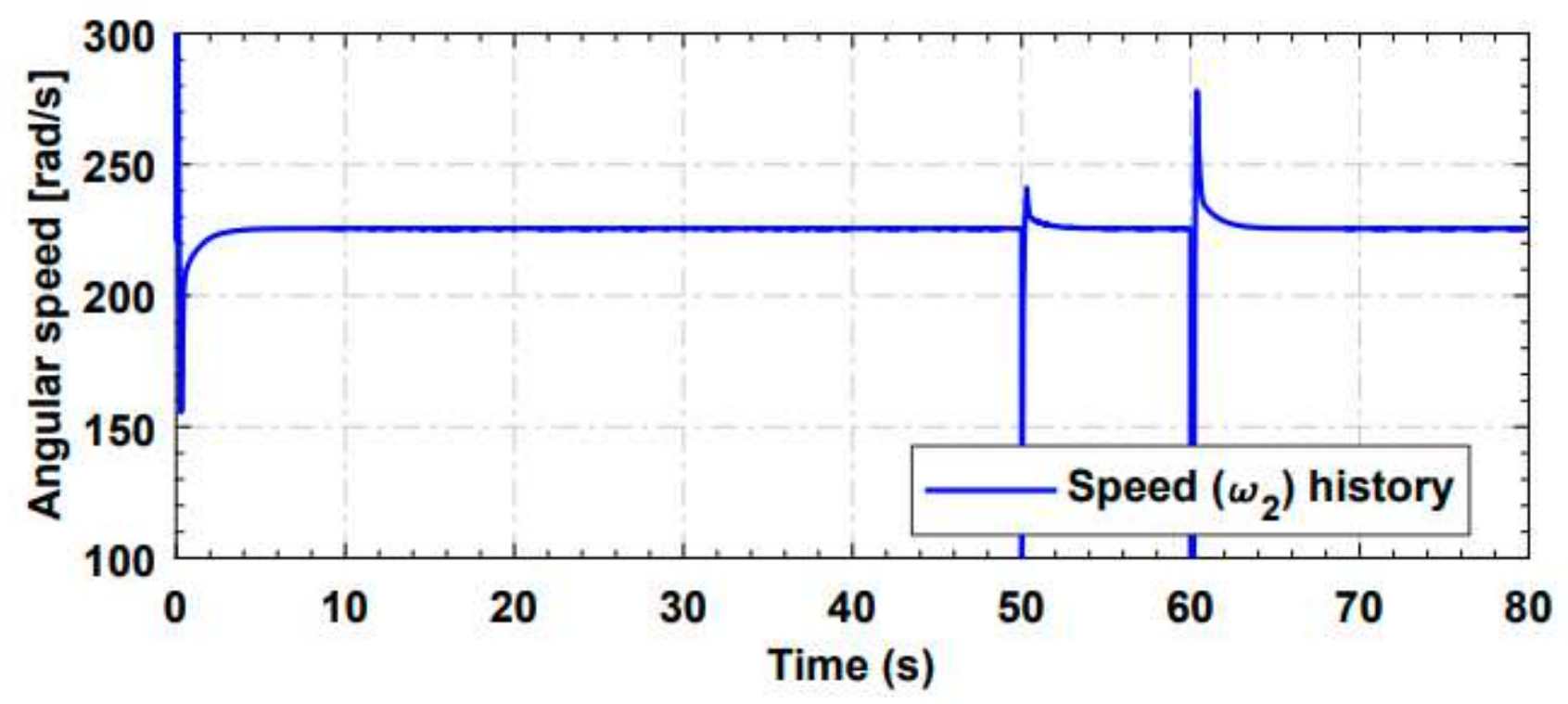

Figure 13

The time history of control input $\omega 2$ produced by the proposed control law

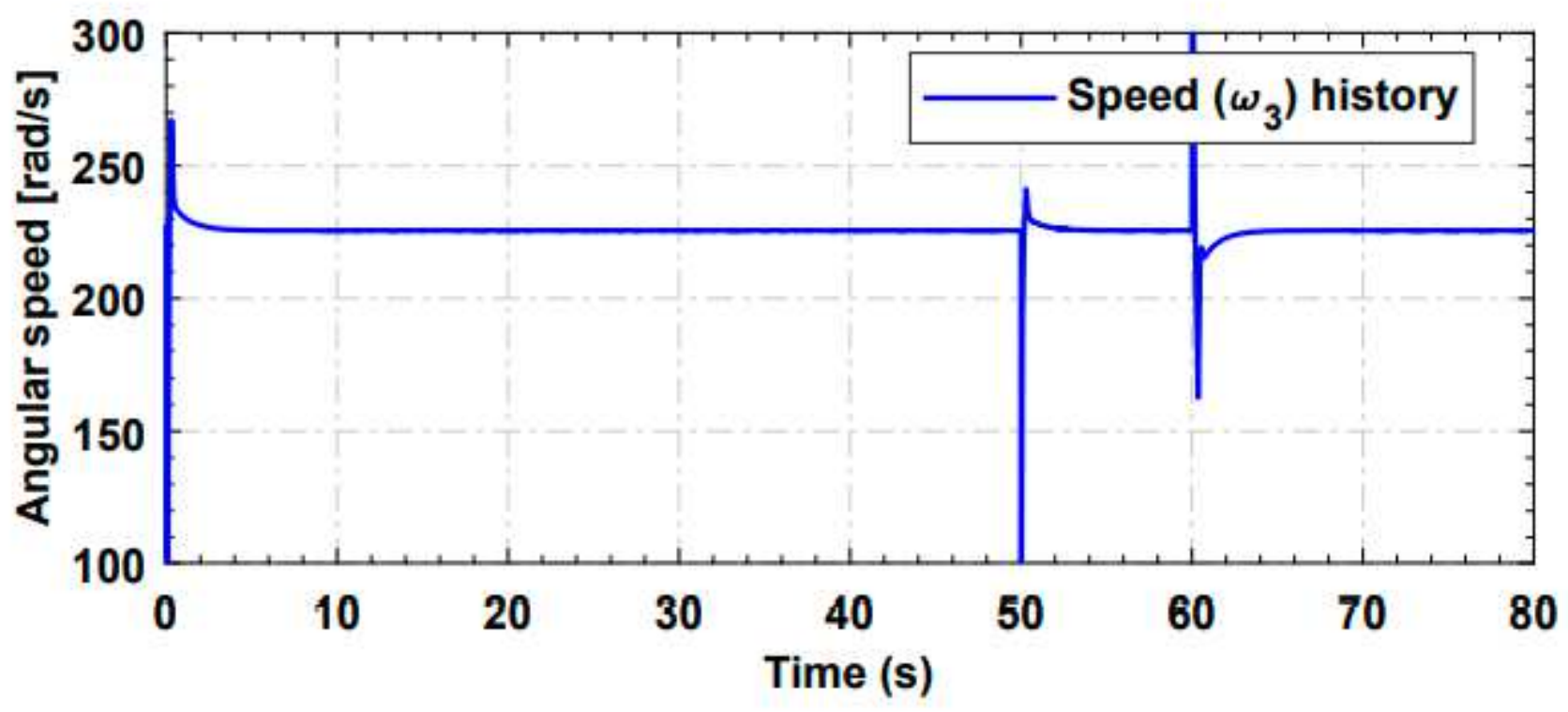

Figure 14

The time history of control input $\omega 3$ produced by the proposed control law 


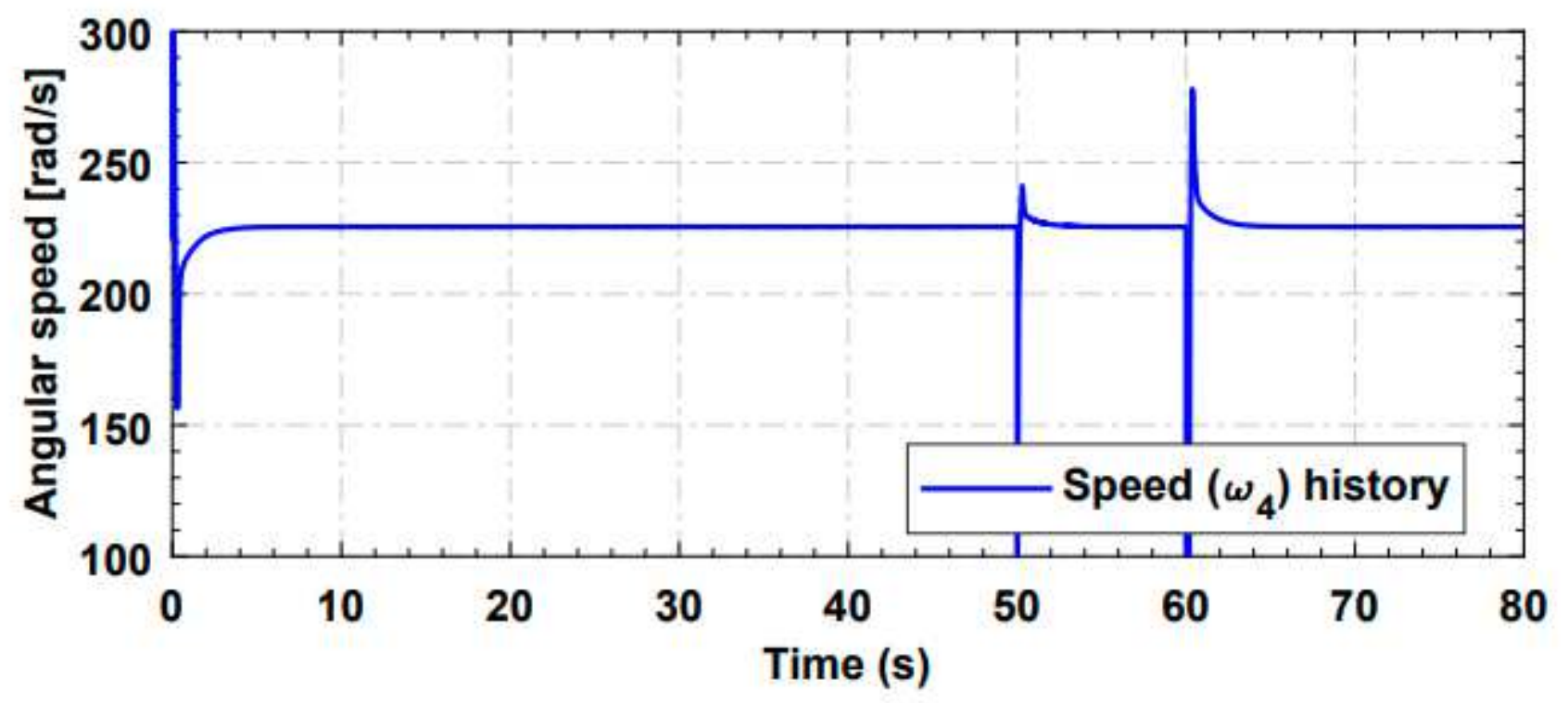

Figure 15

The time history of control input $\omega 4$ produced by the proposed control law 